\title{
Lindahl vs. Lindahl: Optimal siting and sizing of a noxious facility*
}

\author{
Eduardo Ferraz $z^{\dagger}$ and César Mantilla $a^{\ddagger}$
}

March 14, 2021

\begin{abstract}
Providing a noxious facility poses two problems previously unexplored together: where to locate it and how large it should be. We propose a mechanism combining some market-like properties with a modified second-price auction. The mechanism selects a host, a facility size, a compensation for hosting the project, and determines how the compensation and building costs are split among the non-hosts. Regardless of the selected host, any equilibrium outcome of this mechanism is a Lindahl allocation. If each community bids truthfully for becoming the host-a strategy which no community has incentives to deviate-the selected Lindahl allocation is globally optimal.
\end{abstract}

JEL Codes: D61; H40; R53

Keywords: NIMBY; LULU; Lindahl outcomes; public projects; mechanism design

${ }^{*}$ Comments from Diego Aycinena, Lucas Finamor, Ian Flint, Margarita Gáfaro, Vinicius Lima, Pepita Miquel-Florensa, Mounu Prem, Santiago Saavedra and Rodrigo Soares, as well as suggestions from participants in the Economics Seminar at Universidad del Rosario, were extremely valuable. Financial Support from the program "Inclusión productiva y social: programas y politicas para la promoción de una economía formal, código 60185, que conforma la Alianza EFI, bajo el Contrato de Recuperación Contingente No. FP44842-220-2018." is gratefully acknowledged.

${ }^{\dagger}$ Department of Economics, Universidad del Rosario. email: eduardo.ferraz@urosario.edu.co

${ }^{\ddagger}$ Department of Economics, Universidad del Rosario. email: cesar.mantilla@urosario.edu.co 


\section{Introduction}

A noxious facility is a project that serves as a public good for multiple communities, while simultaneously creating a private bad for the hosting community (Kunreuther and Kleindorfer, 1986; Kunreuther et al., 1987). Examples include facilities for waste treatment (e.g., incinerators or disposals), prisons, and wind turbines (Meyerhoff et al., 2010; Zerrahn, 2017). The problems of providing such facilities are described as the "not in my backyard" NIMBY problem, or the "locally unwanted land use" -LULU problem (Popper, 1983; Schively, 2007). Both acronyms make evident that the location problem has attracted more attention than the combined problem of implementing the optimal size and site.

The siting and sizing problems have been separately studied. On the one hand, mechanisms compensating the host of a noxious facility neglect the facility size (Minehart and Neeman, 2002; Sakai, 2012). However, leaving undefined the provided quantity brings as an implicit assumption that the use of the facility is non-rival (Ambec and Kervinio, 2016). On the other hand, some of the existing mechanisms for public goods provision yielding a Lindahl allocation could be employed to determine the optimal size of a noxious facility (Chen, 2002; Van Essen and Walker, 2017). This strand of the literature has paid less attention to the siting problem because, generally, the non-excludability and non-rivalry of public goods reduces the concerns for local effects. This paper aims to explicitly model the endogenous sizing decision jointly with the siting problem in a mechanism that allows for efficient allocations.

Combining the sizing and the siting problem of a noxious facility offers two advantages. First, marginal costs and benefits become central in the normative analysis of the NIMBY problem. Welfare considerations result oversimplified by limiting the collective choice to binary provision decisions. For instance, cost-benefit analyses rarely conclude that a null provision of public bads (e.g., pollution or crime) is optimal. Second, introducing an endogenous facility size allows conceiving this problem in terms of optimally installed capacities, improving future planning.

Lindahl allocations serve as a reference point in the efficient provision of public goods. In a Lindahl allocation, the efficient quantity is attained by charging each community a price proportional to its marginal benefit from this provision-called a Lindahl tax-, granting that the total payments are enough to finance the public good. We extend its use to the provision of a public project, which yields a net benefit for all involved communities but the project host. The extension of this concept to the provision of noxious facilities is natural, with the main difference that the price "charged" to the host has to be negative. Since any community 
can host the facility, we can have as many Lindahl allocations as communities involved in the collective decision. As in the case of public goods, each Lindahl allocation is efficient conditional on the siting, but only one of them is globally efficient.

We propose a mechanism that simultaneously solves the siting problem and the sizing problem of a new facility. Communities involved in the provision of the noxious facility submit a four-dimensional message. Three components interplay in a market-like fashion: a desired size (or quantity) of the facility, a price per unit requested for being the host, and a price per unit paid for being a non-host community. A fourth component, a bid, allows communities to compete for becoming the host in a modified second-price auction.

The market-like components generate Lindahl allocations in equilibrium. If the sum of prices per unit of non-host communities is sufficient to cover the price requested by the host and the building cost per unit, the facility is implemented, and its size is defined by the geometric average of all the desired quantities. Given this design, an equilibrium with implementation implies that: (i) each community submits its Lindahl tax and the total payment matches the total cost and compensation, otherwise each buyer would have incentives to lower its submitted price as buyer; (ii) the outcome quantity is optimal for each community, otherwise the community with a sub-optimal quantity could use the geometric mean to deviate the facility size to its optimal one. Therefore, this mechanism induces Lindahl allocations.

It is precisely because there could be as many Lindahl allocations as communities involved in the mechanism that the market-like part is not enough to find a globally optimal allocation. The siting problem becomes particularly relevant when more than one community would be better off by being the host, relative to being a buyer. In this case, prices cannot be directly used as a selection rule to dictate the hosting community. The reason is that they will create strategic incentives to overstate the willingness to host the facility, distorting the provided quantity: after a competition à la Bertrand, the host will balance the lower compensation per unit of the facility by selecting a size above its optimal.

The auction-like component induces the selection of the globally optimal Lindahl allocation. In our mechanism, each community announces a bid they are willing to pay for becoming the host. The highest bidder is selected and pays the second-highest bid. It may sound counter-intuitive that the community bearing the private costs of the noxious facility has to make a payment. However, this payment acts as a selection device when at least two communities are interested in hosting the facility. This is the reason why the sizing and siting problems cannot be solved sequentially. The intuition is that the community with the 
highest willingness to host the facility is also benefiting the most from not being a buyer. Therefore, no community can profit by misreporting the gains from becoming the host. In this way, as in standard second-price auctions, truthful bidding leads to an efficient allocation even if each community's preferences remain private information, a low informational requirement.

Our mechanism gives a lead role to the sizing problem in the allocation of a noxious facility. By doing so, we can disentangle and prioritize two notions of efficiency. The marketlike part of our mechanism selects, for each specific location, a socially optimal project corresponding to a Lindahl allocation. Thus, efficient sizing is a local property. On the other hand, the auction filters each Lindahl allocation that does not yield the largest facility. Thus, efficient siting is a global property.

Mechanism design has been extensively used to solve the siting problem (Kunreuther et al., 1987; O'Sullivan, 1993; Kleindorfer and Sertel, 1994). Minehart and Neeman's auction (2002) guarantees budget-balancedness, but opens the door to outcomes in which the host is not the community with the lowest willingness to accept a transfer for receiving the facility. Sakai (2012) provides an axiomatic approach to the NIMBY problem, characterizing the fair pricing rules for waste treatment, and showing that Minehart and Neeman's auction does not have a fair pricing rule. Although Minehart and Neeman (2002) and Sakai (2012) introduce some notion of quantity, their set-up leads to a purely siting problem. PerezCastrillo and Wettstein (2002) propose a multi-bidding mechanism where a bid is made for each project. Within the listed projects, the efficient one is selected. The global efficiency of this mechanism relies on including the most socially-desirable project (in size and site) among the listed ones. However, the mechanism is silent on the list elaboration. If this list is short, the inefficiencies arising from project miss-selection tend to be high. As the list becomes longer, the global inefficiency is reduced, but the bid process becomes computationally demanding.

We differ from this literature in two aspects. First, the market-like part of our mechanism transforms the setting from a NIMBY problem into a scenario in which communities actually compete for the hosting role and its associated transfer, defined by prices and provided quantities. Second, whereas most of the previous mechanisms sacrifice efficient host selection for maintaining budget-balancedness, in our mechanism the bid paid by the host is removed from the mechanism's transfers (although it can be reallocated through a non-distortionary rule). The reason for this choice is the importance we give to the pursuit of the globally efficient Lindahl allocation. 
Our model abstains from two aspects complementing the collective nature of the NIMBY problem. First, we do not consider spatial effects in the siting of a noxious facility, implicitly assuming that the facility's environmental costs are encapsulated in the hosting community. Such spatial effects are considered in the mechanisms studied in Waehrer (2003) and Ambec and Kervinio (2016). Abandoning this assumption will increase the complexity of our mechanism, as two different sources of multi-dimensionality must be considered: two prices would result insufficient to capture siting preferences, and the bid would not capture anymore the benefit from switching from being a buyer to be the host (as there would be different "types" of buyers, according to their proximity to the host). Second, we abstain from discussing the aggregation of preferences within a community. ${ }^{1}$ That is, we do not consider the individual incentives behind the delegation process of the community's decision.

The rest of this paper goes as follows. Section 2 presents a comparison with relevant mechanisms. Section 3 defines a Lindahl outcome for the problem of a noxious facility, its efficiency properties, and explains why we might have multiple Lindahl outcomes. Section 4 explains the functioning of the mechanism. Section 5 describes how the mechanism selects Lindahl outcomes and the filtering conditions for reaching the globally optimal Lindahl allocation. Since we departed from the usual approach of sacrificing efficient host selection to grant budget balancedness (from the community's perspective), Section 6 is devoted to discussing the efficiency properties of our mechanism. In Section 7, we provide a discussion on whether some desirable properties apply to our mechanism. We show in Section 8 that our mechanism is robust to generalizations in communities' preferences.

\section{A comparative analysis with existing mechanisms}

In this section, we argue that, although the literature on public choice with transferable payoffs is large and mature, the existing mechanisms cannot provide feasible solutions to the siting and sizing problems. We divide our argument into three parts. First, we discuss why VCG-type of mechanism performs poorly when dealing with noxious facilities. Second, we compare our informational requirements with those from other mechanisms efficiently solving the siting problem. Finally, we explain why the assumptions of some previous mechanisms lead to a pure reallocative problem, whereas we also consider the technological transformation caused by the noxious facility.

At first sight, it might sound appealing to use the VCG mechanism to solve the NIMBY

\footnotetext{
${ }^{1}$ See the Appendix of Minehart and Neeman (2002) for an axiomatic solution to this problem.
} 
problem: conditional on participation, the VCG provides the optimal allocation of the facility. However, participation in the mechanism is precisely what makes the VGC unsuitable for the NIMBY problem. Recall that the VCG mechanism charges each participant a price equal to the caused externality. The price for each community results from computing the differences between the welfare of the other communities under the most efficient allocation, with and without the community's utility.

In the NIMBY problem, if the host's utility were ignored as part of the welfare, the social planner would not account for the host's private costs from building the facility in its land. Since non-hosts are better off with a larger facility, the planner would opt for an exceedingly large size. Therefore, the externality caused by the host is the utility the other communities lose due to a facility's size reduction, making the Clarke-tax for the host necessarily positive. Consequently, communities with low costs for hosting the facility-the most socially-desirable hosts-would be discouraged to participate. This leads to inefficient outcomes, if not to the absence of participants. By contrast, any equilibrium in our mechanism is Pareto improving and does not harm participation.

Our framework also relies on realistic informational assumptions. We only require the facility's financial cost to be public information, with preferences being private knowledge. This contrasts with Perez-Castrillo and Wettstein (2002) and Laurent-Lucchetti and Leroux (2011). Pérez-Castrillo and Wettstein's mechanism is efficient, budget-balanced, and it allows introducing spatial effects into the NIMBY problem. Still, it comes at the cost of assuming individual preferences to be common knowledge. Laurent-Lucchetti and Leroux propose a mechanism in which the most potential host is selected in a first stage, but it is excluded from a second stage in which other communities submit their contributions. ${ }^{2}$ The mechanism's sequential nature reduces the strategic incentives associated with role uncertainty, as our auction does, but their informational requirement, much weaker than in Pérez-Castrillo and Wettstein's, is yet too stringent: all the communities are ex ante aware of who is the most efficient host.

Compared to reference papers imposing informational requirements similar to ours, our paper differs in the nature of the social gains caused by the noxious facility. For instance, the concavity in the facility's cost depending on its size (O'Sullivan, 1993) or in the disutility caused by the noxious good (Minehart and Neeman, 2002; Sakai, 2012), turns the provision

\footnotetext{
${ }^{2}$ In a companion paper, Laurent-Lucchetti and Leroux (2010) propose a Lindahl taxation scheme, but they assume a specific project and location. This initial effort to connect the notion of Lindahl pricing and the NIMBY problem fell short in acknowledging that the social benefits from implementation critically depend on both the plant size and which community is selected as the host.
} 
of a noxious facility into a reallocative problem. The main consequence is that, in both cases, with property rights over the noxious good, a market could solve this problem equivalent to finding the "right" host. For some intuition, imagine there is a community $i$ with the lowest marginal willingness to accept a unit of the noxious good, given by $p$. If the market defines a price slightly above $p$ for accepting each unit of the good, all communities are better off when $i$ "stores" the total amount of the good.

We rather focus on the class of noxious goods causing convex private costs. ${ }^{3}$ Here, the technological transformation of the noxious good is the determinant of efficiency. By selecting an optimal size, the communities use the mechanism to jointly select an installed capacity able to transform a given amount of the noxious good into a by-product that has a lower monetary equivalent in terms of disutility. Hence, an additional source of gains, apart from reallocative efficiency, dwells on the transformation of the noxious good into a "cheaper" source of disutility.

\section{Framework}

We consider a set $N=\{1,2, \ldots, n\}$ of at least three communities, all involved in a one-time collective decision of providing a noxious facility $Z$ of variable size. The marginal financial cost of the facility is $c$, which we assume to be common knowledge. The facility's size captures the amount of output the facility can produce: its power capacity if $Z$ is a thermal power station, or its waste processing capacity if $Z$ is an incinerator, to name a couple of examples.

Communities are interested in the benefits of using a common facility. Given the noxious nature of this facility, the benefits are globally enjoyed by all the non-host communities, and the environmental costs are locally borne by the host. Thus, we introduce $v_{i}$ to represent the valuation of the facility size for non-hosts and for the host community. We define $v_{i}(Q)$ as $i$ 's willingness to pay to enjoy, as a non-host, the benefits of a facility with size $Q$. In addition, $i$ 's willingness to accept hosting this facility is $-v_{i}(-Q)$ (so $v_{i}(-Q)$ is negative). We set $v_{i}(0)=0$ to represent the status quo when no facility is provided. Before jumping into the assumptions we make about $v_{i}$, we discuss the meaning of this function informally.

\footnotetext{
${ }^{3}$ Imagine (i) a prison that, the larger it is, the higher the profile of prisoners or the higher the likelihood of inner gangs; or (ii) a nuclear power plant in which the concerns about safety increase disproportionately faster than its energy-producing capacity.
} 


\subsection{On the interpretation of $v_{i}$}

We introduced $v_{i}$ with the classic approach of a non-rival good (Ambec and Kervinio, 2016), even though the nature of the noxious facility suggests that its output is rival. However, our use of $Q$ is easily reconciled with the private use of $Z$ by the non-host and host communities. We will give an example of how private interests to use a rival share of the facility can be expressed in terms of a common $Q$.

Suppose the facility built in some community has a size $Q$, that its ownership is well defined, and that there is a competitive market to trade its output. Let $w_{i}(\rho)$ be the community $i$ 's willingness to pay to use $\rho$ units of $Z$. Assume that $p_{Z}$ is the market price of the output of $Z$, so $i$ seeks to maximize $w_{i}(\rho)-p_{Z} \rho$ in $\rho$. This problem yields a solution $\rho_{i}$ such that $w_{i}^{\prime}\left(\rho_{i}\right)=p_{Z}$. Since this holds for each community, it is easy to see that $\rho_{i}$ and $p_{Z}$ depend only on $Q$, so we write $\rho_{i}=\rho_{i}(Q)$ and $p_{Z}=p_{Z}(Q)$. If the marginal returns with $Z$ 's output are decreasing, a larger size $Q$ implies a lower price $p_{z}$, so the indirect utility $v_{i}(Q)$ that a non-host $i$ derives from this facility of size $Q$ is the function $w_{i}\left(q_{i}(Q)\right)-p_{Z}(Q) q_{i}(Q)$, increasing in $Q$.

In plain words, when the preferences and market structure are given, the indirect utility of communities can only depend on the economic fundamental $Q$. Thus, the benefits of a larger facility mirror the gains from trade in a market with less scarcity of goods.

Note that community $i$ serving as the host also receives the benefit described in the previous paragraph. Nonetheless, its net benefit also includes the indirect (dis)utility of hosting the facility of size $Q$. Thus, $v_{i}(-Q)$ is equal to $v_{i}(Q)$ minus its willingness to accept the losses caused by hosting $Z$. We assume that $v_{i}$ is negative in its negative domain. This assumption justifies why communities do not merely build the facility autarchically but rather profit from building it collectively.

As it is standard in this literature, we parametrize the function $v_{i}$ as $d_{i} v$, where $d_{i}$ is a positive constant for each community. This imposes a symmetry on the benefits and the costs of the facility: if a community enjoys more the gains of being a non-host, it also bears a greater cost of being the host.

This condition is way less restrictive than it looks. First, this may capture a high correlation between benefits and costs for some noxious facilities. The reason is that the benefits from non-hosting the facility can be interpreted as the foregone costs of the status quo solution: more pristine communities, willing to pay more for implementing the facility elsewhere, are also more affected if they end up being the host, even though in both scenarios they de- 
sire implementation (e.g., waste incinerators). ${ }^{4}$ Second, the correlation between benefits and costs may result strengthened by other heterogeneities: in the spirit of Olson's argument (1965), wealthier or larger communities may simultaneously have a higher willingness to pay (for non-hosting) and to accept (for hosting), as is reflected in their larger weight in the collective decision at the expense of a larger burden.

Moreover, in Section 8, we show that the hypothesis of symmetry between costs and benefits is not even necessary. We stick to this hypothesis because it allows us to report our procedures more concisely, while it can also be used as a reference to show the functioning of our mechanism under much less restrictive assumptions. More specifically, all the results of this paper hold if there is a community $i_{s}$, which we call the suitable host, such that $v_{i_{s}}^{\prime}=\min _{i}\left\{v_{i}^{\prime}\right\}$ and $v_{i_{s}}^{\prime \prime}=\min _{i}\left\{v_{i}^{\prime \prime}\right\}$. We also consider a case where $d$ is replaced by two parameters, breaking the symmetry between costs (as host) and benefits (as non-host). Although we find that there are parameter configurations where the mechanism does not provide an efficient outcome, simulations suggest that these configurations are unlikely to occur.

\subsection{Assumptions about the preferences}

As stated in Section 3.1, we assume that $v_{i}=d_{i} v$. Now, we define the properties of the function $v$. We let $v: \mathbb{R} \rightarrow \mathbb{R}$ be a strictly increasing function respecting $v(0)=0$, $v^{\prime \prime}<0, v^{\prime \prime \prime} \geq 0, \lim _{x \rightarrow \infty} v(x)=\infty, \lim _{x \rightarrow-\infty} v^{\prime}(x)=\infty$, and $\lim _{x \rightarrow \infty} v^{\prime}(x)=0$. The asymptotic assumptions, together with the existence of $v^{\prime}(0)$, are useful to focus on wellbehaved outcomes in the following sections. Nonetheless, these assumptions are primarily technicalities and can be dropped without harming the results. In addition, $v^{\prime \prime}(0)$ and $v^{\prime \prime \prime}(0)$ do not need to be well defined.

Following the discussion of the previous section, we assume that the utility of community $i$ is additively separable and linear in money. If the provision of $Z$ for community $i$ is $q$-which is negative if $i$ is the host-and $i$ receives a transfer $T$, then its utility, defined as $u_{i}(q, T)$, is given by $d_{i} v(q)+T .{ }^{5}$ Communities with low values of $d$ are good candidates for becoming the host since they are more comfortable accepting larger facilities in their backyard in exchange for a smaller transfer, compared to cities with a larger value of $d$. The $d$ 's are assumed to be

\footnotetext{
${ }^{4} \mathrm{~A}$ counter-example would be wind turbines, where the benefits (cleaner energy) are poorly correlated with the costs (landscape deterioration).

${ }^{5}$ Throughout this paper, we generally use the uppercase $Q$ to denote physical quantities of the facility, which are positive, and the lowercase $q$ to denote chosen quantities, which will be negative for the host community.
} 
private information and without loss of generality we set $d_{1}<d_{2}<\cdots<d_{n}$.

\subsection{Lindahl outcomes}

Lindahl outcomes are key in our framework. As we show in this section, we can mirror the standard Lindahl efficiency properties from the provision of public goods in the context of noxious facilities. These properties ultimately drive the design of our mechanism.

Suppose that $Z$ is offered to community $i$ at a price $p$. In this case, $i$ is interested in the provision of $q$ units of $Z$, where $q$ respects $d_{i} v^{\prime}(q)=p$. The preferences for the facility siting (i.e., whether community $i$ prefers to be the host or a buyer) depend on the relationship of the offered price $p_{i}$ with respect to $\bar{p}_{i}:=d_{i} v^{\prime}(0)$, which we call the no-trade price of community $i$. We thus have a null demand $(q=0)$ if $p=\bar{p}_{i}$; a demand for buying, with $q>0$, if $p<\bar{p}_{i}$; and a demand for hosting, with $q<0$, if $p>\bar{p}_{i}$. Figure 1 illustrates these three cases. Notice from panel (a) that the utility is negative whenever the community is a buyer (resp. the host) under a price greater (resp. smaller) than $\bar{p}$. Panel (b) depicts the "demand" curve for $Z$, remarking a switch in the desired role of a community in $\bar{p}$.

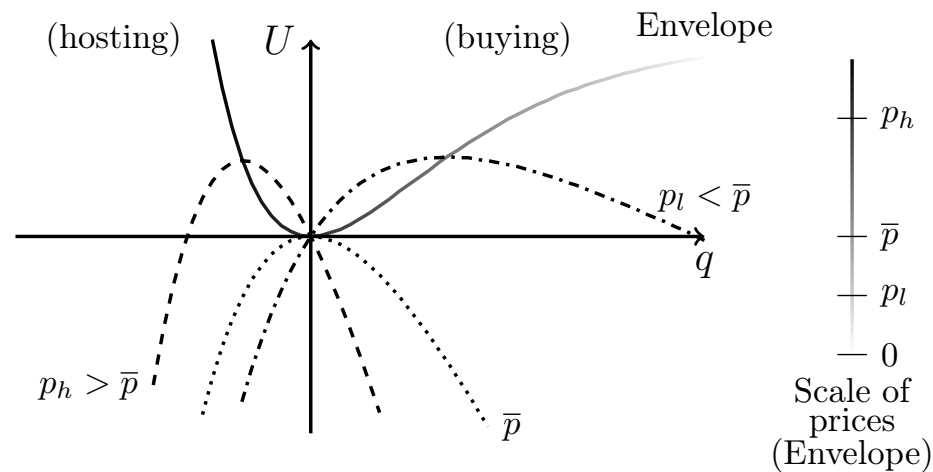

(a)

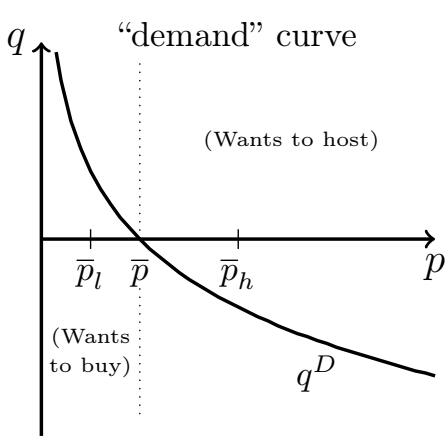

(b)

Figure 1: The no-trade price is $\bar{p}$, and we have $p_{l}<\bar{p}<p_{h}$. (a) Utility level as a function of the size of $Z$ for the three prices $p_{h}, \bar{p}$, and $p_{l}$. Each point of the Envelope curve represents the optimal quantity and the corresponding utility value for a given price. (b) The "demand" curve for $Z, q^{D}$. Notice that, after $\bar{p}$, there is a demand for negative quantities that translates into an acceptance to host.

For some $\boldsymbol{p}=\left(p_{1}, p_{2}, \ldots, p_{n}\right), Q>0$, and $j \in N$, the triplet $(j, Q, \boldsymbol{p})$ is a Lindahl outcome if it respects three conditions: (i) for each $i \in N \backslash\{j\}, i$ pays $p_{i}$ per unit of $Z$, and $Q$ is the optimal quantity for $i$ under $p_{i}$; (ii) $j$ receives $p_{j}$ per unit of $Z$ as a host and $Q$ is the optimal hosted quantity for $j$ under $p_{j}$; (iii) the buyers' total payment matches the payment asked by the host plus the cost of implementation of $Z$. That is, $\sum_{i \neq j} p_{i}=p_{j}+c$. 
We describe the conditions under which a Lindahl outcome $(j, Q, \boldsymbol{p})$ exists. Since $Q$ is the optimal quantity for each community under $\boldsymbol{p}$, we have $d_{i} v^{\prime}(Q)=p_{i}$ for each $i \neq j$, and $d_{j} v^{\prime}(-Q)=p_{j}$. Applying these first-order conditions into the budget balancedness condition gives us

$$
\frac{v^{\prime}(-Q)}{v^{\prime}(Q)}=\frac{\sum_{i \neq j} d_{i}}{d_{j}}-\frac{c}{d_{j} v^{\prime}(Q)} .
$$

This implies that community $j$ is a potential host in a Lindahl outcome if, and only if, it belongs to the set

$$
L_{I}:=\left\{k \in N \mid d_{k}<\sum_{i \neq k} d_{i}-c / v^{\prime}(0)\right\},
$$

where $L_{I}$ stands for "Lindahl-implementable". The solution of Equation 1 is unique in $Q$ for each $j$. Therefore, when the solution is positive, we call Lindahl $j$ the Lindahl outcome when $j$ is the host. We define it as $\left(j, Q^{L_{j}}, \boldsymbol{p}^{L_{j}}\right)$, with $\boldsymbol{p}^{L_{j}}=\left(p_{1}^{L_{j}}, \ldots, p_{n}^{L_{j}}\right)$. In addition, we call the pair $\left(j, Q^{L_{j}}\right)$ a Lindahl- $j$ allocation.

From the definition of $L_{I}$, there might exist up to $n$ Lindahl outcomes, depending on the parameter $c$ and the $d$ 's. For some intuition, communities with a low $d$ are more likely to belong to the set $L_{I}$ because they are more willing to accept a transfer in exchange for hosting the facility. Nonetheless, this set can be empty if the marginal cost $c$ is too large relative to the values of $d$. Conversely, a null marginal cost ensures that at least Lindahl-1 exists. Figure 2 presents an example in which three communities can generate three Lindahl outcomes. Note that the "demand" curves for the three communities are identical across panels. In panel (a), under the price $p_{1}^{L_{1}}$, community 1 has a negative demand for $Z$, while communities 2 and 3 have a positive demand under prices $p_{2}^{L_{1}}$ and $p_{3}^{L_{1}}$, respectively. Besides, $Q^{L_{1}}$ is the optimal size of $Z$ for each community under $\boldsymbol{p}^{L_{1}}$, and we have $p_{1}^{L_{1}}=p_{2}^{L_{1}}+p_{3}^{L_{1}}$. The reasoning is similar for panels (b) and (c), with communities 2 and 3 acting as hosts. Notice that $Q^{L_{1}}>Q^{L_{2}}>Q^{L_{3}}$.

We now turn to the efficiency properties of Lindahl outcomes. Fix an arbitrary $j \in N$. Consider the social planner problem under the monetized welfare metric constrained to implement $Z$ in community $j$ and to a balanced budget. The social planner maximizes

$$
v(x) \sum_{i \neq j} d_{i}+d_{j} v(-x)+c x
$$

in $x$ subject to $x \geq 0$. If $j \notin L_{I}$, then we have a corner solution. The social planner's solution is the status quo, which in this case is also Pareto optimal. By contrast, if we have $j \in L_{I}$, the first-order condition for the optimal size $Q$ is given by Equation 1. That is, the planner's 


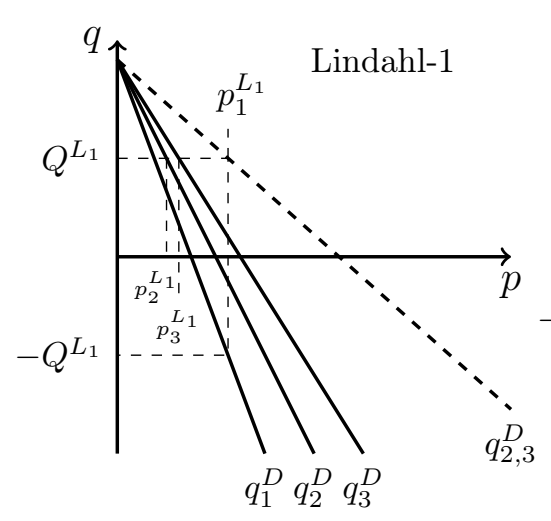

(a)

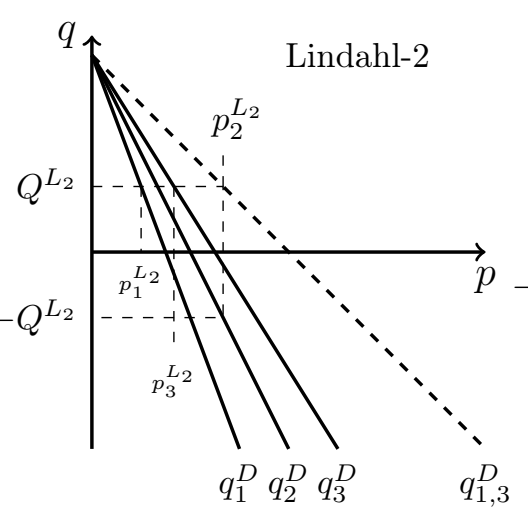

(b)

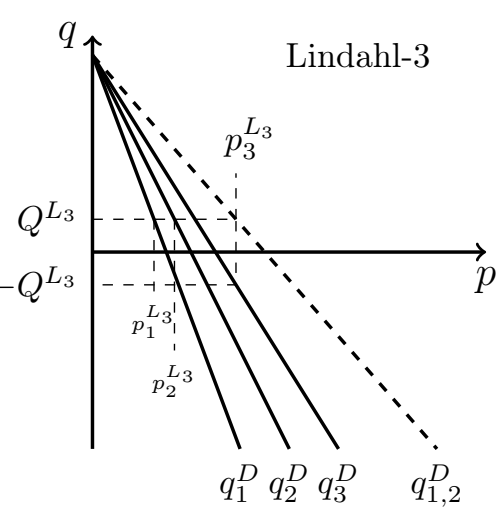

(c)

Figure 2: The "demand" curve of community $i$ is $q_{i}^{D}$ and the aggregate demand of communities $i$ and $j$ is $q_{i, j}^{D}$. The building cost is 0 . (a) Lindahl-1 outcome. (b) Lindahl-2 outcome. (c) Lindahl-3 outcome.

solution is the Lindahl- $j$ allocation. Since, in addition, Lindahl $j$ is a Pareto improvement from the status quo, we conclude that Lindahl $j$ is Pareto optimal for a fixed $j .{ }^{6}$

We just described the planner's solutions under the restriction of installing $Z$ in $j$-yielding standard results from the literature. We now analyze the global solution of the planner, relaxing the location constraint. As a departing point, we argue that $Q^{L_{j}}$ is decreasing in $j$. To see why, fix a size for $Z$. Because the social planner's problem must be budget balanced (i.e., the sum of transfers cancels out), the social surplus from the implementation of $Z$ can be assessed by weighing the costs and benefits of the facility. While the marginal private cost for community $j$ is increasing in $j$, the total marginal private benefit for the set of communities $N \backslash\{j\}$ is decreasing in $j$. This implies that the net marginal surplus is decreasing in $j$. Since the marginal social surplus is zero at $Q^{L_{j}}$ in Lindahl- $j$, it has to be positive at $Q^{L_{j}}$ in Lindahl- $(j-1)$, that is, $Q^{L_{j}}<Q^{L_{j-1}}{ }^{7}$

Therefore, as we move from Lindahl $-j$ to Lindahl- $(j-1)$, we can break down the welfare change into two components: a larger surplus per unit of $Z$ up to $Q^{L_{j}}$; plus an increase in the installed capacity from $Q^{L_{j}}$ to $Q^{L_{j-1}}$. We thus conclude that Lindahl-1 is socially optimal.

Proposition 1. Suppose $L_{I}$ is non-empty. Then Lindahl-1 is welfare-maximizing, and the Lindahl-1 allocation is the only one yielding an optimal facility size.

This proposition ensures Lindahl-1 is Pareto optimal, but it does not tell us anything

\footnotetext{
${ }^{6}$ To see why Lindahl- $j$ Pareto dominates the status quo, recall that $u_{i}\left(0,0 p_{i}^{L_{j}}\right)=0$, and that $Q^{L_{j}}>0$ maximizes the utility of each community under Lindahl-j prices. Hence, $u_{i}\left(Q^{L_{j}}, Q^{L_{j}} p_{i}^{L_{j}}\right)>u_{i}\left(0,0 p_{i}^{L_{j}}\right)$ for $i \neq j$. The same holds for community $j$ exchanging $Q^{L_{j}}$ by $-Q^{L_{j}}$.

${ }^{7}$ By contrast, host prices $p_{j}^{L_{j}}$ and host transfers $p_{j}^{L_{j}} Q^{L_{j}}$ are not necessarily monotonic on $j$.
} 
about Pareto dominance between Lindahl outcomes. Indeed, provided that $j \in L_{I} \backslash\{1\}$, each community in $N \backslash\{j\}$ benefits more from Lindahl- 1 than from Lindahl $-j .{ }^{8}$ But since community 1 prefers Lindahl- 1 to Lindahl $-j$, if $d_{j}$ is close enough to $d_{1}$ and they virtually share the same preferences, community $j$ is better off as the host in Lindahl- $j$ compared to being a buyer in Lindahl-1. This allows us to conclude that Lindahl-1 is a Pareto improvement with respect to Lindahl- $j$ only when $d_{j}$ is sufficiently larger than $d_{1}$.

Since the social surplus is maximized at Lindahl-1, the social planner would prefer a Lindahl-1 allocation, followed by any budget balanced redistribution of resources, compared to a Lindahl- $j$ allocation. However, to achieve this first best, the informational requirement that the $d$ 's are public-or, at least, known by the social planner-is too stringent. The following section presents a mechanism granting the most efficient siting and sizing, even if the $d$ 's are treated as private information.

\section{The mechanism}

We now introduce the proposed multi-dimensional mechanism. Each community $i$ submits a four-element message: the price as host $p_{i}^{h}$ (in practice, the marginal willingness to accept the project), the price as buyer $p_{i}^{b}$ (in practice, the marginal willingness to pay for the project), a desired quantity $q_{i}$, and a bid $b_{i}$.

Although the multi-dimensional strategies are simultaneously submitted, we provide a first glimpse of how the mechanism operates by following the sequential outputs it yields. First, the community with the highest bid is selected as the host and its submitted price as host becomes its operating price for the rest of the mechanism. Similarly, for each other community, its operating price corresponds to the submitted price as buyer. The project is implemented only if the aggregate marginal willingness to pay-the sum of all buyers' operating prices-meets the host's marginal willingness to accept (plus the marginal cost of the facility's construction). In case that the former equals the latter, the individual trade prices-a mechanism's output defining the unit price of the facility for each community-are identical to the operating prices. A simple rebate rule adjusts the individual trade prices when there is an excess contribution. Figure 3 illustrates how these arguments operate in the case of three cities.

A reader may wonder whether the four elements are necessary. They are. Think sepa-

\footnotetext{
${ }^{8}$ We use the negative concavity of $v$ and the monotonicity of $Q^{L_{j}}$ to show that a community in $N \backslash\{1, j\}$ is better off in Lindahl-1. Lemma 4 shows the same for community 1.
} 


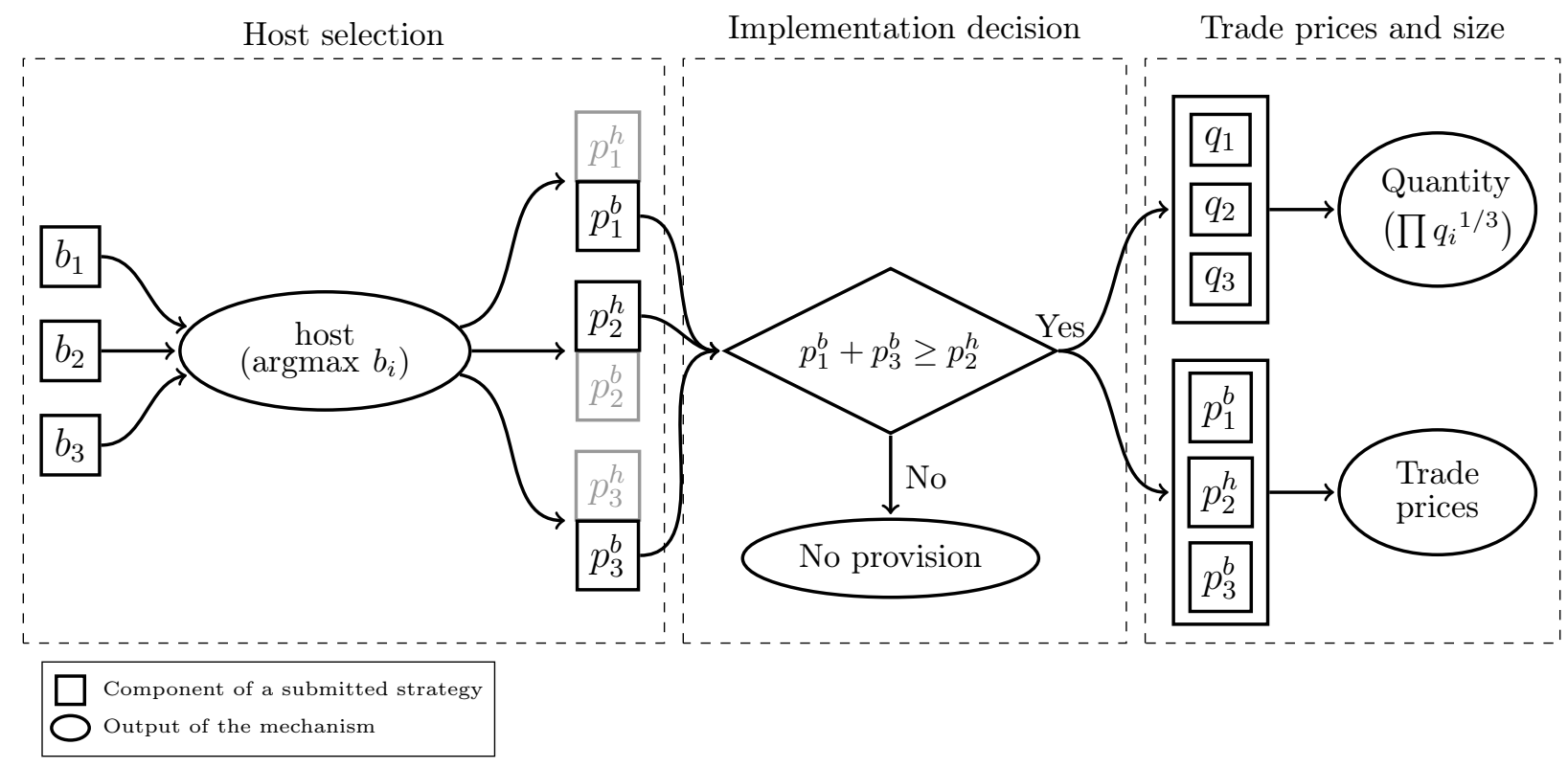

Figure 3: An illustration of the mechanism with communities 1, 2, and 3, with no building cost, when 2 sets the highest bid. The sequential outputs of the mechanism are observed in the three dashed boxes when read from left to right. On the leftmost box, bids define the host as well as the operating prices $p_{1}^{b}, p_{2}^{h}$, and $p_{3}^{b}$. The central box verifies whether the sum of 1's and 3's prices as buyer meets the price as host of 2 to grant implementation. The rightmost box displays the provided quantity and trade prices.

rately on the sizing and siting problems. Deciding how large the facility should be, and how much each community will have to pay for its use, implies communities submitting a price and a quantity. Deciding the facility's host will require a bid per community interacting in an auction-like mechanism, adding a third element. The fourth one, an additional price, allows connecting both problems while guaranteeing the global efficiency described in Section 3.3.

Formally, let $\Omega:=\mathbb{R}_{++} \times \mathbb{R}_{+}^{3}$ be the space of strategies. For any strategy profile $s \in \Omega^{n}$, the mechanism $\mathcal{M}$ maps $s$ onto: 1. a host $\hat{i}, 2$. the provision level of $Z, \hat{Q}$, and 3 . the financial transfer $\hat{T}_{i}$ made by community $i$, which can be positive or negative. ${ }^{9}$ More specifically, let $\boldsymbol{s}=\left(s_{1}, s_{2}, \ldots, s_{n}\right)$ where $s_{i}=\left(q_{i}, p_{i}^{b}, p_{i}^{h}, b_{i}\right)$ is the strategy of community $i \in N$. The outcomes are defined as follows.

Host selection The community with the highest bid is selected as host. If there is a tie, the first tie-breaking criterion is the lowest price as host. A draw is used as a second tie-break

\footnotetext{
${ }^{9}$ As a convention, variables with a hat are outcomes of the mechanism for a strategy profile $\boldsymbol{s}$. When the context is clear, we omit the argument $\boldsymbol{s}$. This abuse of notation causes no confusion in the text.
} 
criterion. ${ }^{10}$ We denote the host as $\hat{i}$. We define the community with the second-highest bid (following the same tie-break rules if necessary) as $\hat{j}$. Since each community submits two prices but is assigned to a single role, half of these prices are discarded. With the definition of the host $\hat{i}$, the prices $p_{i}^{h}$ for $i \neq \hat{i}$ and $p_{\hat{i}}^{b}$ are denoted the residual prices because they do not enter into the mechanism's computations. By contrast, we call $p_{\hat{i}}^{h}$ and $p_{i}^{b}$ for $i \neq \hat{i}$ the operating prices.

Provision We start by defining the excess contribution $\hat{\pi}$ as the difference between the aggregate marginal willingness $\sum_{i \neq \hat{i}} p_{i}^{b}$ and the marginal costs-the host's compensation $p_{\hat{i}}^{h}$ plus the physical construction $c$. That is, $\hat{\pi}:=\sum_{i \neq \hat{i}} p_{i}^{b}-c-p_{\hat{i}}^{h}$. The facility is implemented when the excess contribution is non-negative. We call this condition the implementation rule. When this condition is respected, the facility size $\hat{Q}$ is given by the geometric mean of the submitted quantities. That is, $\hat{Q}=\prod_{i \in N}\left(q_{i}\right)^{1 / n_{1}} \mathbb{1}_{\{\hat{\pi} \geq 0\}}$.

Since $q_{i}>0$ for each $i$, communities do not have veto power via submitted quantities. Moreover, the geometric mean ensures that no player can cap, from above or below, the implemented quantity. Therefore, when $Z$ is implemented in equilibrium, $\hat{Q}$ has to be optimal for each community.

Transfers First, we define the prices communities will actually pay (or receive, in the host's case) per unit implemented of the project, which we call the trade prices. These prices are given by the operating prices, compensated by a share of the excess contribution. The share of each buyer is $\frac{1}{2(n-1)}$. Thus, if community $i$ is a buyer, its trade price is given by $\hat{p}_{i}^{b}:=p_{i}^{b}-\frac{\hat{\pi}}{2(n-1)}$ and $i$ receives the (negative) transfer $\hat{T}_{i}:=-\hat{Q} \hat{p}_{i}^{b}$.

In the same way, the host's trade price is $\hat{p}:=p_{\hat{i}}^{h}+\hat{\pi} / 2$ so that $\hat{i}$ receives from the other communities $\hat{Q} \hat{p}$. Note that, whereas the share of each buyer is $\frac{1}{2(n-1)}$, the host's share is half of the excess contribution, so the total amount paid by the buyers is received by the host. ${ }^{11}$ The host also bears two potential costs: the host $\hat{i}$ pays the second-highest bid, $b_{\hat{j}}$, in case of implementation; and a penalty $r>0$ in case of no implementation. Therefore, the net transfer received by the host $\hat{i}$ is given by $\hat{T}_{\hat{i}}=\hat{Q} \hat{p}-b_{\hat{j}} \mathbb{1}_{\{\hat{\pi} \geq 0\}}-r \mathbb{1}_{\{\hat{\pi}<0\}}$. Figure 4 depicts the interplay between the operating prices and the excess contribution, yielding the trade prices for each community.

\footnotetext{
${ }^{10}$ While the first tie-break criterion helps for the efficient selection of the host, the only purpose of the second one is to define a unique host for any given strategy profile.

${ }^{11}$ As long as each community has a positive share of $\hat{\pi}$, the exact split of the excess contribution does not alter the mechanism's results.
} 


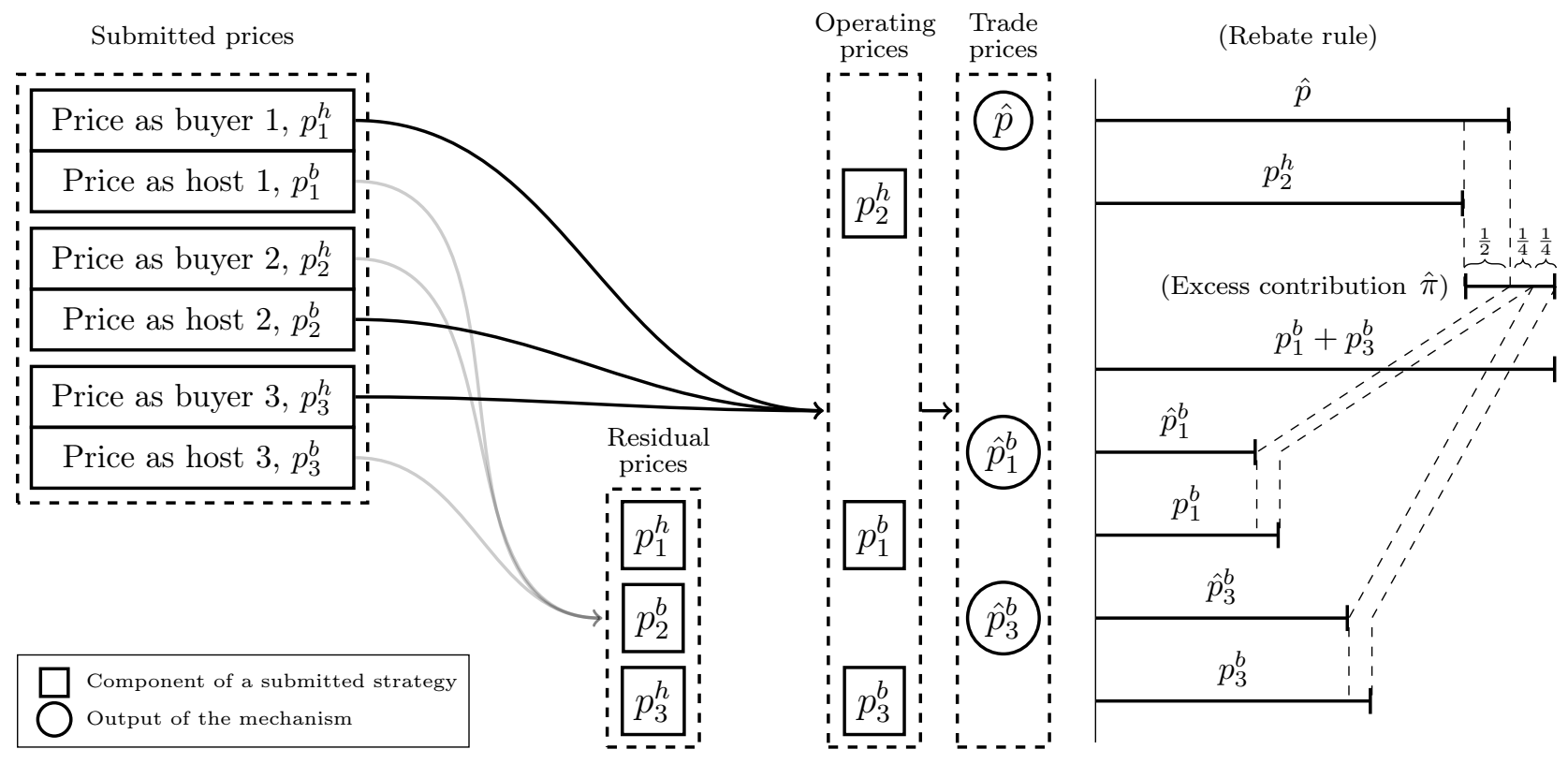

Figure 4: An illustration of how submitted prices are divided into residual and operating prices, and how the latter define trade prices using a simple rebate rule. We continue with the example from Figure 3: communities 1,2 , and 3 , no building cost, and 2 setting the highest bid.

Having defined how the mechanism operates, we focus now on two key features of it: its analogy to a multi-step mechanism and why (and when) it is not budget balanced. Figure 3 depicts a sequential interpretation of the mechanism, despite the simultaneous nature of submitted strategies. Following this depiction, we provide a more detailed description of the outcomes in a backward induction-manner.

In its "second" step, the mechanism operates as a market. The intended contributions as non-hosts, and the requested compensations as hosts, interplay in the form of prices per unit of $Z, p^{b}$ and $p^{h}$. In case of "trade," the mechanism defines mutually agreed prices and quantities and establishes a vector of prices $\hat{\boldsymbol{p}}$ per unit of $Z$. This vector contains a price $\hat{p}_{i}^{b}$ for each buyer $i$ and a price $\hat{p}$ for the host. We define $\tilde{\mathcal{M}}:=(\hat{i}, \hat{Q}, \hat{\boldsymbol{p}})$ as the "pre-taxation" outcome of the mechanism. It is the budget-balanced outcome of $\mathcal{M}$ before the payment of the bid $b_{\hat{j}}$ or the penalty $r$.

The "initial" step of the mechanism works like an auction, in which each community submits a bid. The community selected as the host pays the second-highest bid, emulating how a second-price auction selects the most efficient bidder. Although it may sound counterintuitive that the host must pay a bid for installing a facility that creates environmental costs for itself, this payment is required to select the most efficient siting. The intuition is that the 
bid intends to capture the community's willingness to pay to switch from being a buyer to become the host, given the expected positive transfer $\hat{Q} \hat{p}$ based on the prices and quantities defined in the second, market-like, step. Not surprisingly, the community with the lowest $d$ is in a position to make the highest bid. We explain this idea now with further details.

Suppose that, for some given opponents' strategies, the payoff for community $i$ of being the host is $g_{i}^{h}>0$, while the payoff of being a buyer is $g_{i}^{b}>0$. Suppose, in addition, that $i$ prefers to be the host. Therefore, $i$ 's willingness to pay to change its role from a buyer to the host is positive and given by $\Delta_{i}:=g_{i}^{h}-g_{i}^{b}$. The condition $\Delta_{i}>0$ typically reveals that $d_{i}$ is low compared to the other $d$ 's. By the definition of willingness to pay, $i$ does not have incentives to bid more than $\Delta_{i}$. At the same time, bidding less than $\Delta_{i}$ means that $i$ might miss the opportunity of changing roles, even when the cost of doing so is lower than $\Delta_{i}$. Therefore, $\Delta_{i}$ is a natural candidate for $i$ 's bid.

Now consider a community $j$ challenging $i$ 's willing of being the host, and define $g_{j}^{h}, g_{j}^{b}$, and $\Delta_{j}$ analogously. The relation between the private costs communities $i$ and $j$ have for hosting the project depends on whether $d_{j}$ is larger or smaller than $d_{i}$. If $d_{j}<d_{i}$, then, due to the lower private cost of $j$ to host $Z$, we have $g_{j}^{h}>g_{i}^{h}$; otherwise, $g_{j}^{h}<g_{i}^{h}$. Similarly, a lower $d$ leads to a higher $\Delta$, since this community results better off by being the host and not a buyer. Hence, if communities are truthfully bidding their $\Delta$ 's, the community with the lowest $d$ ends up being the host. In other words, communities with higher $d$ drop out first from the race for hosting because they reach earlier a bid equal to $\Delta$.

Thus, the project is implemented in the community with the lowest private cost of hosting it. The remaining $n-1$ communities, those with the highest value from having the facility elsewhere, end up as buyers. In other words, our mechanism pays one cost, the bid, for suppressing the cost of misallocating the host. However, note that the second-highest bid, $b_{\hat{j}}$, will be positive only when the lowest parameters are sufficiently close, so both are interested in becoming the host. Otherwise, $b_{\hat{j}}$ will be zero, preserving the budget balancedness of the outcome.

We conclude this section explaining the role of the last component of the mechanism, the penalty $r$, imposed on the host when there is no project implementation. Suppose that, for some given opponents' strategies, $i$ is the host and its payoff for implementing any level of $Z$ is negative. The penalty $r$ eliminates the emergence of the following equilibrium: $i$ vetoing the implementation of $Z$ by raising its price as host until the excess contribution becomes negative. No matter how small $r$ is, community $i$ is better off providing a sufficiently small facility, or changing its bid to become a buyer, than paying $r$. Since any Lindahl outcome 
Pareto dominates the status quo, $r$ is efficiency-enhancing provided that $L_{I}$ is non-empty.

\section{Equilibrium analysis}

For the sake of clarity, we will assume in this section that $L_{I}$ is non-empty. That is, we focus on parameter configurations guaranteeing the existence of at least one Lindahl outcome.

Before moving to the functioning of equilibria with provision, let us explain when $L_{I}$ is non-empty. Consider two communities 1 and $a$, where $a$ is the aggregation of communities $2, \ldots, n$; and with $\bar{p}_{1}$ and $\bar{p}_{a}$ being their no-trade prices. Recall that the no-trade price is the infimum (resp. supremum) of the prices under which a community would accept to host (resp. buy) a positive quantity of $Z$. The condition $L_{I} \neq \emptyset$ is equivalent to $c<\bar{p}_{a}-\bar{p}_{1}$, where $\bar{p}_{a}=\sum_{i=2}^{n} \bar{p}_{i}$. In other words, that there exists some size of $Z$ such that "community" $a$ would accept to pay for the facility and adequately compensate the host. ${ }^{12}$

We study equilibria implications of the mechanism $\mathcal{M}$ under the traditional definition of ex post equilibria. For each $i \in N$ and a strategy profile $s=\left(s_{1}, \ldots, s_{n}\right) \in \Omega^{n}$, we define the strategy-based utility of community $i$ as

$$
U_{i}(\boldsymbol{s})=d_{i} v(-\hat{Q}(\boldsymbol{s})) \mathbb{1}_{\{i=\hat{i}(\boldsymbol{s})\}}+d_{i} v(\hat{Q}(\boldsymbol{s})) \mathbb{1}_{\{i \neq \hat{i}(\boldsymbol{s})\}}+\hat{T}_{i}(\boldsymbol{s}) .
$$

In addition, we define $\boldsymbol{s}_{-i}:=\left(s_{1}, \ldots, s_{i-1}, s_{i+1}, \ldots, s_{n}\right)$ and denote $\boldsymbol{s}=\left(s_{i} ; \boldsymbol{s}_{-i}\right)$. We say that $s$ is an equilibrium if, for each $i$, there is no $\tilde{s}_{i}$ such that $U_{i}\left(\tilde{s}_{i} ; \boldsymbol{s}_{-i}\right)>U_{i}\left(s_{i} ; \boldsymbol{s}_{-i}\right)$.

The first equilibrium result we establish is that our mechanism induces Lindahl outcomes before the bid payment.

Proposition 2 (Necessity). Suppose that $\boldsymbol{s}$ is an equilibrium. Then the prices and quantities obtained from $\tilde{\mathcal{M}}(\boldsymbol{s})$ correspond to a Lindahl outcome, and $\mathcal{M}(\boldsymbol{s})$ Pareto dominates the status quo.

Notice that there are three main statements in this proposition. First, an equilibrium implies project implementation. This is a direct consequence of the penalty $r$ imposed on the

\footnotetext{
${ }^{12}$ Restating a model hypothesis and rewriting two points of the mechanism, it is possible to incorporate an equilibrium with no implementation when $L_{I}=\emptyset$. Suppose that, for $\epsilon>0, v$ is linear in $[-\epsilon, \epsilon]$. Consider a modification to the mechanism in which the implemented quantity is the minimum between the geometric mean and $\epsilon$. Besides, in case of no implementation, instead of punishing $\hat{i}$ with $r$, each community pays a penalty equal to $-\hat{\pi} \epsilon$. This induces communities to implement a quantity $\epsilon$, in equilibrium, when $L_{I}$ is empty. Since we can make $\epsilon$ arbitrarily small, this can be virtually turned to no implementation.
} 
host under no implementation. We describe the idea to prove this feature of the mechanism in the concluding lines of Section 4.

Second, $\mathcal{M}(s)$ improves the outcome for each community with respect to the status quo. Since the mechanism aggregates quantities geometrically, if $U_{i}(\boldsymbol{s})<0$, community $i$ could reduce the provision level of $Z$, contradicting the fact that $s$ is an equilibrium. In other words, the mechanism is individually rational in equilibrium. Third, the pre-taxation mechanism outcome $\tilde{\mathcal{M}}(\boldsymbol{s})$ is necessarily a Lindahl outcome. The implementation rule implies that $p_{\hat{i}}^{h} \leq \sum_{i \neq i} p_{i}^{b}-c$, but if the inequality were strict $\hat{i}$ would have incentives to increase its price as host. Satisfying $p_{\hat{i}}^{h}=\sum_{i \neq \hat{i}} p_{i}^{b}-c$ means that, in order not to jeopardize provision, communities effectively act as price-takers. Given the geometric aggregation of quantities under our mechanism, $\hat{Q}$ must be simultaneously optimal for each community for such given prices. Hence, the selected prices reflect an optimal quantity for each community, implying that all conditions of a Lindahl outcome are met.

Proposition 2 characterizes the equilibria, but it does not tell us anything about their existence. The next proposition shows that the set of equilibria is non-empty. To lighten the notation, we define, for any $s \in \Omega^{n}$ and each $i \in N, \bar{b}_{i}\left(\boldsymbol{s}_{-i}\right):=\max \left\{b_{j}: j \in N \backslash\{i\}\right\}$,

$$
\begin{gathered}
g_{i}^{b}\left(\boldsymbol{s}_{-i}\right):=\sup \left\{U_{i}\left(s, \boldsymbol{s}_{-i}\right): s \in \Omega \text { and } i \text { is a buyer }\right\}, \\
g_{i}^{h}\left(\boldsymbol{s}_{-i}\right):=\sup \left\{U_{i}\left(s, \boldsymbol{s}_{-i}\right)+\bar{b}_{i}\left(\boldsymbol{s}_{-i}\right): s \in \Omega \text { and } i \text { is the host }\right\},
\end{gathered}
$$

and $\Delta_{i}\left(\boldsymbol{s}_{-i}\right):=g_{i}^{h}\left(\boldsymbol{s}_{-i}\right)-g_{i}^{b}\left(\boldsymbol{s}_{-i}\right)$. Therefore, loosely speaking, for a given $\boldsymbol{s}_{-i}, g_{i}^{h}\left(\boldsymbol{s}_{-i}\right)$ is the best outcome that community $i$ can get as the host, and $g_{i}^{b}\left(\boldsymbol{s}_{-i}\right)$ the best outcome that community $i$ can get as a buyer, prior to the bid payment. The quantity $\Delta_{i}\left(\boldsymbol{s}_{-i}\right)$ reflects the willingness to pay of community $i$ to change from being a buyer to become the host, for a given opponent's profile. We call $\Delta_{i}\left(\boldsymbol{s}_{-i}\right) \mathbb{1}_{\Delta_{i}\left(\boldsymbol{s}_{-i}\right) \geq 0}$ the switching valuation, since this is the bid that $i$ is willing to offer to switch roles (i.e., from buyer to host). Note that the switching valuation is a function of $\boldsymbol{s}_{-i}$.

Proposition 3. Let $\boldsymbol{s}^{\star}$ be the strategy profile, depending only on the fundamentals (d's and c):

(i) the submitted quantities are all $Q^{L_{1}}$;

(ii) the submitted prices satisfy $p_{1}^{h}=p_{1}^{L_{1}}, p_{1}^{b}=d_{1} v^{\prime}\left(Q^{L_{1}}\right)$, and, for every $i \geq 2, p_{i}^{b}=p_{i}^{L_{1}}$ and $p_{i}^{h}=\max \left\{p_{1}^{L_{1}}-p_{i}^{L_{1}}+p_{1}^{b}, d_{i} v^{\prime}(0)\right\}$;

(iii) the submitted bids satisfy $b_{i}=\Delta_{i}\left(\boldsymbol{s}_{-i}^{\star}\right) \mathbb{1}_{\left\{\Delta_{i}\left(\boldsymbol{s}_{-i}^{\star}\right) \geq 0\right\}}$ for each $i \in N$. 
Then, $\boldsymbol{s}^{\star}$ is an equilibrium and $\tilde{\mathcal{M}}\left(s^{\star}\right)$ is Lindahl-1. Moreover, this equilibrium is robust to any coalition of communities in the set $N \backslash\{1\}$.

Here is an alternative way of understanding this statement. Consider an equilibrium $\boldsymbol{s}^{\star}$ in which community 1 submits its best prices as buyer and host under a quantity $Q^{L_{1}}$, and bids its switching valuation. This switching valuation is high enough to make it too costly for any other community to become a host. Thus, the best that communities $2, \ldots, n$ can do is to maximize their payoffs as non-hosts by submitting Lindahl-1 prices as buyers. In particular, since $\Delta_{j}\left(s^{\star}\right)<\Delta_{1}\left(s^{\star}\right)$ for $j \geq 2$, bidding their switching valuations ensures they end up as buyers. ${ }^{13}$ Only a Lindahl-1 can emerge from this simultaneous maximization and, therefore, $\tilde{\mathcal{M}}\left(s^{\star}\right)$ is Lindahl-1.

This result agrees with the intuition. Since $d_{1}$ is the lowest, community 1 bears the lowest cost from hosting $Z$ and it would have received a lower benefit as a buyer. Thus, it is expected that community 1 would be able to pay the highest value to take the role of host.

It turns out that under $\boldsymbol{s}^{\star}$, whereas communities $2, \ldots, n$ extract from the mechanism a surplus proportional to its prices as buyers, the surplus extracted by community 1 is more than proportional to its residual (buying) price. Let $u_{0}:=v\left(Q^{L_{1}}\right) / v^{\prime}\left(Q^{L_{1}}\right)-Q^{L_{1}}$. The value $p_{i}^{b} u_{0}$ is the monetized benefit of community $i \geq 2$. For community 1 , being a buyer would have yielded a benefit $p_{1}^{b} u_{0}=g_{1}^{b}\left(\boldsymbol{s}^{\star}\right)$. The upper bound of $i$ 's payment as the host is its own bid. Thus, $g_{1}^{h}\left(\boldsymbol{s}^{\star}\right)=g_{1}^{b}\left(\boldsymbol{s}^{\star}\right)+\Delta_{1}\left(\boldsymbol{s}^{\star}\right) \geq p_{1}^{b} u_{0}$ confirms that community 1 is favored by $\mathcal{M}\left(\boldsymbol{s}^{\star}\right)$ in the sense that it receives a disproportionately higher benefit, compared to its price as buyer. $^{14}$

Proposition 3 also says that the host's privileged position cannot be successfully challenged by any coalition of the buyers defined by $\boldsymbol{s}^{\star}$. Suppose that communities $i$ and $j$, in the role of buyers, form a coalition and assign $j$ to be the host. This coalition cannot profit from the margins: in order to secure implementation, any increase in the price as host submitted by $j$ has to be compensated with an equal decrease in the price as buyer submitted by $i$. Changing quantities will not help the coalition either. If a quantity $\tilde{Q}$ could increase the surplus of $\{j, i\}$, then $\tilde{Q}$ could improve the situation of at least one of them individually, which contradicts $\boldsymbol{s}^{\star}$ being an equilibrium. A similar idea applies if $i$ and $j$ try to increase their surplus in a coalition where both are buyers.

The most technical issue in the computation of equilibria comes from accounting possible deviations from buyer communities-in which residual prices play an important role. For

\footnotetext{
${ }^{13}$ We show that $\Delta_{j}\left(s^{\star}\right)<\Delta_{1}\left(\boldsymbol{s}^{\star}\right)$ for $j \geq 2$ in the proof of Proposition 3 .

${ }^{14}$ Since $p_{i}^{b} u_{0}=d_{i} v^{\prime}\left(Q^{L_{1}}\right) u_{0}$, we can show in the same lines that community 1 is the only receiving a benefit that is proportionally greater than its parameter $d$.
} 
instance, suppose that $d_{j}$ is close to $d_{1}$, and that community 1 submits 0 as its price as buyer. This could trigger a scenario in which $j$ is better off as a host (before the bid payment), and 1 prefers being a buyer with a null price instead of being a host under a price $p_{1}^{L_{1}}$. In this case, a Lindahl-1 outcome for $\tilde{\mathcal{M}}$ in equilibrium could not be sustained. What we show in Proposition 3 is that there is at least one price as buyer for community 1 sustaining a Lindahl-1 outcome.

We thus guarantee not only the existence of an equilibrium, but the one yielding the most efficient facility size. However, the previous propositions do not answer what happens if, following the discussion above, community $j \geq 2$ behaves as the host and every other community selects their optimal prices, or contributions, with respect to $Q^{L_{j}}$. The next proposition states that, if each community bids its switching valuation, then Lindahl- $j$ cannot be an equilibrium.

Proposition 4. If the submitted bids satisfy $b_{i}=\Delta_{i}\left(\boldsymbol{s}_{-i}\right) \mathbb{1}_{\left\{\Delta_{i}\left(\boldsymbol{s}_{-i}\right) \geq 0\right\}}$ for each $i \in N$, then there is no $s \in \Omega^{n}$ such that $\tilde{\mathcal{M}}(\boldsymbol{s})$ is Lindahl-j for $j \geq 2$.

If a community decreases its price as host, it creates stronger incentives for other communities to be buyers. Therefore, a priori, a community $j \geq 2$ could try to use its price as buyer as an instrument to hold its position as the host. Proposition 4 says that, as long as other communities bid their switching valuation, whenever $j$ 's price as buyer sustains its position as the host, $j$ would rather be a buyer-no matter how close $d_{j}$ is to $d_{1}$.

Figure 5 gives some intuition on the two previous propositions. Let $y$ and $x$ be the two communities with the lowest $d$ 's. Each curve corresponds to the difference between the switching valuations of communities $y$ and $x$ as a function of the price as buyer that community $y$ will set, $p_{y}^{b}$. If $y$ is the host, the mechanism provides a Lindahl- $y$ allocation. Otherwise, the price $p_{y}^{b}$ is such that $x$ submits the best price as host to adhere to the implementation rule. By moving to the upper curves, we consider scenarios in which $d_{y}$ decreases, holding $d_{x}$ constant. The two top curves exemplify Proposition 3, applying when $d_{y}<d_{x}$. Community $y$ ensures having a higher switching valuation by submitting a price $d_{y} v^{\prime}\left(Q^{L_{y}}\right)$ as host (see points $A$ and $B$ on the $d_{y} v^{\prime}\left(Q^{L_{y}}\right)$ curve). This price (but also any other price ensuring $y$ a higher switching valuation) would discourage $x$ from submitting a bid large enough to become the host, since the latter community will be better off as a buyer.

The two bottom curves illustrate Proposition 4, applying when $d_{y}>d_{x}$. Even if $y$ submits a price as buyer that maximizes $\Delta_{y}-\Delta_{x}$ (see points $C$ and $D$ ), this difference is negative. Therefore, if $x$ and $y$ truthfully bid their switching valuations, $x$ will submit the highest bid and $Z$ is not built in $y$. Note also that when the communities are identical, with $d_{y}=d_{x}$, 
the maximum of $\Delta_{y}-\Delta_{x}$ equalizes their switching valuations precisely at the intersection with the $d_{y} v^{\prime}\left(Q^{L_{y}}\right)$ curve (see point $E$ ). Hence, two symmetric communities competing for hosting the facility will dissipate all the rents obtained from holding this role.

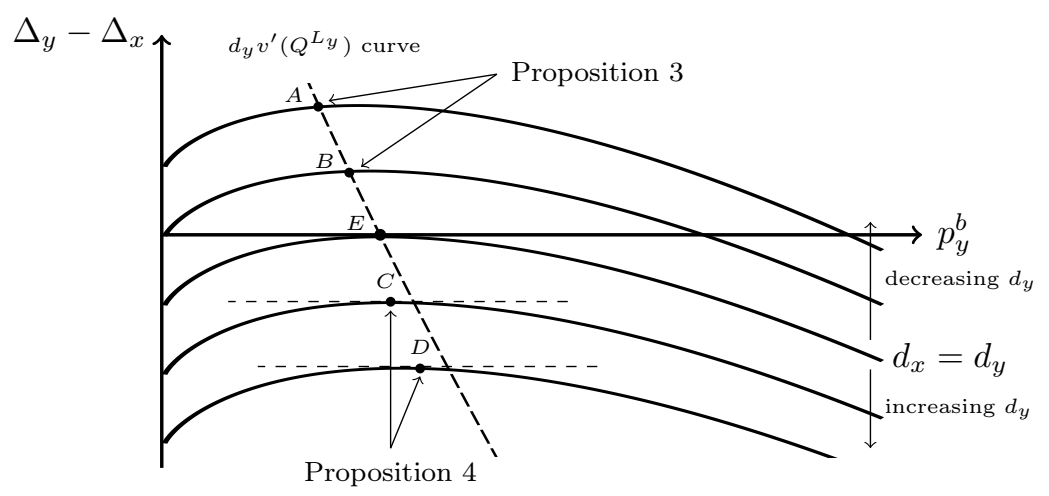

Figure 5: Competition on switching valuations between two communities.

To explain the bidding restriction we impose on Proposition 4, we turn ourselves once again to the analogy between a standard second-price auction and the mechanism $\mathcal{M}$. In a second-price auction, even in equilibrium, any bidder with positive valuation can be the winner. The well-known result, yielding the bidder with the highest valuation as the unique possible winner, emerges only after refining equilibria to dominant strategies (Jackson and Swinkels, 2005). Bidding the switching valuations in our mechanism does not perfectly parallel bidding valuations in second-price auction. In particular, our mechanism does not allow for dominant strategies. Nonetheless, bidding truthfully in our proposed mechanism shares four features with the second-price auction. First, it is a transparent and straightforward strategy. ${ }^{15}$ Second, it is the only bid from which, regardless of the opponents' bids, communities do not have incentives to deviate. ${ }^{16}$ Third, the equilibrium allocation of bidding truthfully is unique, depending only on the parameters. Fourth, as we discuss in detail in the following section, this type of bid leads to efficiency.

\footnotetext{
${ }^{15}$ Minehart and Neeman (2002) employ the same adjectives to describe why understating the community's disutility from hosting the project involves some risk, while bidding "close" to the true disutility allow communities to "do well" in different environments.

${ }^{16}$ To see why, consider a strategy profile $\boldsymbol{s}$ with $g_{i}^{h}\left(\boldsymbol{s}_{-i}\right)>g_{i}^{b}\left(\boldsymbol{s}_{-i}\right)$. Community $i$ would be better off as the host whenever $g_{i}^{h}\left(\boldsymbol{s}_{-i}\right)-\bar{b}_{i}\left(\boldsymbol{s}_{-i}\right)>g_{i}^{b}\left(\boldsymbol{s}_{-i}\right)$, or $\boldsymbol{\Delta}_{i}\left(\boldsymbol{s}_{-i}\right)>\bar{b}_{i}\left(\boldsymbol{s}_{-i}\right)$. In the same way, $i$ would strictly prefer being a buyer when $\Delta_{i}\left(s_{-i}\right)<\bar{b}_{i}\left(s_{-i}\right)$. Thus, bidding $\Delta_{i}\left(s_{-i}\right)$ always puts $i$ in its best role between buyer and host, regardless of $\bar{b}_{i}\left(s_{-i}\right)$-even when opponents are not bidding their switching valuations.
} 


\section{$6 \quad$ Efficiency analysis}

Before discussing our mechanism's efficiency properties, we want to raise a point about the two types of inefficiencies existing in mechanisms for locating noxious facilities. The first type is due to an inefficient choice of a siting of the facility. The other type, the one potentially arising in our mechanism, comes from budget unbalancedness. To avoid distortions in incentives, mechanisms collecting a surplus cannot rebate these excess transfers to the communities. Therefore, from the perspective of the communities, this surplus is a financial loss.

We argue that these inefficiencies are not equivalent. Inefficient siting causes a deadweight loss analogous to a misallocation problem. On the other hand, when there is budget unbalancedness, the surplus can be collected by an external agent, an "auctioneer."

The main result of this section, a corollary of propositions 1 to 4, states that our mechanism induces the implementation of a noxious facility that is efficient in both size and site. Recall that Proposition 1 tells us that Lindahl-1 is welfare-maximizing, while Proposition 3 grants that our mechanism can generate the Lindahl-1 allocation in equilibrium. Moreover, Proposition 2 rules out as equilibrium any allocation that is not a Lindahl one, and Propostition 4 eliminates Lindahl- $j$ allocations for $j \geq 2$ as equilibrium, as long as communities bid their switching valuations. This is enough to prove the following theorem.

Theorem 5. If the submitted bids satisfy $b_{i}=\Delta_{i}\left(\boldsymbol{s}_{-i}\right) \mathbb{1}_{\left\{\Delta_{i}\left(\boldsymbol{s}_{-i}\right) \geq 0\right\}}$ for each $i \in N$, then the unique equilibrium allocation $\mathcal{M}$ allows is the efficient one.

Unlike most of the existing mechanisms for locating noxious facilities, we opted to grant an efficient quantity in equilibrium at the cost of having a non-deficitary budget unbalancedness. We have already argued that a surplus on transfers is, in a sense, a weaker form of inefficiency than a deadweight loss. Another reason for our choice, holding even if the bid payment is seen as a deadweight loss, is that moving from a Lindahl- $j$ to a Lindahl-1 allocation simultaneously improves siting and sizing. Previous papers have accounted for the efficiency gains from moving the project to communities having lower private costs for hosting the facility. By explicitly modeling quantities as a decision variable, we add another dimension of efficiency: since the average unit of $Z$ becomes less costly for society, better locations also grant welfare improvements from larger projects.

This theorem also lets us discuss some natural limits for this efficiency loss. On the one hand, the maximum loss is limited to $\Delta_{1}(\boldsymbol{s})$ for any $\boldsymbol{s}$ sustaining a Lindahl-1. To see why, recall that as $d_{2}$ approaches to $d_{1}$, the switching valuation of community 2 approaches to the 
one of community 1. Since we assume communities are bidding their switching valuations, the bid community 1 pays can be arbitrarily close to $\Delta_{1}(s) \cdot{ }^{17}$

On the other hand, the minimum loss is zero. There is a threshold value $\bar{d}$, depending only on $d_{1}$, such that, when $d_{2}>\bar{d}$, the bid paid by the host is zero. Intuitively, the auction embedded in our mechanism serves the purpose of allowing potential hosts to compete. Having $d_{2}>\bar{d}$ implies that, putting community 1 aside, no community can profit more as the host than as a buyer, even before the bid payment. Therefore, all communities except for 1 will bid zero, and the host role of community 1 is not contested. Consequently, our mechanism reaches the first best, a Lindahl-1 with budget balancedness, when $d_{2}>\bar{d}$.

\section{Other properties of the mechanism}

To conclude, we now discuss some properties of the mechanism regarding individual rationality, fairness, and robustness to coalitions.

\subsection{Individual rationality}

Since the host could always deviate to become a buyer, and a null price as buyer grants a non-negative payoff, all equilibria (weakly) Pareto dominate the status quo. Moreover, as a consequence of Propositions 1 and 2, each buyer ends up with a strictly positive payoff in any equilibrium.

Out of equilibrium, the problem is more convoluted. Communities in the role of buyers can secure a null payoff, but the host cannot. However, if no community wants to host the facility, all communities face the risk of taking this undesired role: even if communities submit a null bid and a high price as host (the first tie-breaking criterion), all other communities might bid likewise, with even higher hosting prices.

A possible issue is that, for any given strategy, a community might suffer unbounded losses within the mechanism. Restricting the support of the parameter space so for each $i \in N$ we have $d_{i} \in\left[d_{\min }, d_{\max }\right]$, and capping the provision of $Z$ by a quantity $\bar{Q} \geq Q^{L_{1}}$ can limit the loss to the penalty $r$, while keeping its efficiency properties. ${ }^{18}$

With this cap, community $i$ could submit a null bid and a price $d_{i} v^{\prime}(-\bar{Q})$ as host. Under

\footnotetext{
${ }^{17}$ In particular, if communities use the strategy formulated in Proposition 3, we can express the switching valuation of community 1 as $d_{1}\left(v\left(-Q^{L_{1}}\right)-v\left(Q^{L_{1}}\right)+Q^{L_{1}}\left(v^{\prime}\left(-Q^{L_{1}}\right)-v^{\prime}\left(Q^{L_{1}}\right)\right)\right)$. This expression depends only on the $d$ 's and on the cost $c$, since $Q^{L_{1}}$ is a direct function of them.

${ }^{18}$ The quantity $\bar{Q}$ solving $d_{\min } v^{\prime}(-\bar{Q})+c=(n-1) d_{\max } v^{\prime}(\bar{Q})$ respects this condition.
} 
this strategy, the trade price for $i$ is at least $d_{i} v^{\prime}(-\bar{Q})$, and any quantity that $i$ may provide dwells between 0 and $\bar{Q}$, giving $i$ a positive payoff. In the worst-case scenario for the host community, it pays $r$ for not implementing the facility. However, this penalty can be made arbitrarily small without harming the efficiency properties of $\mathcal{M}$. If we allow for the possibility of not implementing the facility in equilibrium, then we can set $r=0$ in the mechanism and obtain the property of ex post individual rationality.

\section{$7.2 \quad$ Fairness}

Recall from Section 5 that the monetized benefits among buyers are proportional to the price each community pays as a buyer under $\boldsymbol{s}^{\star}$. This property, inherited from Lindahl outcomes due to Proposition 2, actually holds for all equilibria in $\mathcal{M}$. The proportionality between payments for, and benefits from, the provided facility encompasses the following notion of fairness among buyers: the more communities contribute, the more they benefit. Our fairness notion is different from the one proposed in Sakai (2012). In his case, fairness

relates to proportional compensations: communities representing heavier burdens to the welfare receive lower benefits from the mechanism.

In Section 5, we also conclude that the strategy $\boldsymbol{s}^{\star}$ favors community 1 . This host remains favored by the mechanism, for strategies different to $\boldsymbol{s}^{\star}$, under two conditions: when $d_{2}$ is not too close to $d_{1}$; and, if this condition does not hold, when the (buying) residual price of the host falls below its residual price under $\boldsymbol{s}^{\star}$. However, it is not possible to describe a rule in which the host's benefits are always disproportionately large with respect to buyers. Hence, our fairness analysis is limited when it applies to the host.

\subsection{Robustness to coalitions}

In Proposition 3, we show that the Lindahl-1 equilibrium is robust to coalitions of communities in the role of buyers. By contrast, coalitions between the host and a buyer with a sufficiently low $d$ can alter the mechanism's outcome. Nonetheless, and unlike most cases in which collusive agreements might result detrimental to society, any coalition involving community 1 is, in fact, efficiency-enhancing.

Consider the following example. Community 2 agrees to bid $\Delta_{2}-K$ if community 1 makes a side payment of $K / 2$ after being selected as the host. Note that, as $K$ increases, the bid paid by community 1 becomes smaller, reducing the surplus that makes our mechanism budget unbalanced. Hence, coalitions including community 1 are efficiency-enhancing because they 
reduce the competition for hosting the facility; and since $\Delta_{1}>\Delta_{j}$ for $j \geq 2$, communities in the coalition will pursue the Lindahl-1 allocation because it maximizes such side-payments.

Intuitively, the larger is the coalition including community 1 , the lower the screening costs in our second-price auction, and therefore the bid paid by the host decreases. For instance, in a coalition of communities 1 and 2, the former will pay at most $b_{3}$ as host. Applying this argument iteratively, if all communities with $\Delta>0$ are part of the coalition, the costly screening is no longer necessary. Nonetheless, the market-like part of our mechanism will perform efficiently.

\section{Robustness to non-parametric $v_{i}$}

So far, we have presented the results of the mechanism when the heterogeneity between communities is generated by the parameter $d$. Its simplicity provides a better idea of how the mechanism selects communities with lower $d$ 's, which are explicitly linked with host selection and, therefore, efficiency. This section discusses how the mechanism performs under a much broader class of functions representing the indirect utilities.

Consider a non-parametrized set of increasing and concave indirect utilities $v_{i}$ for $i \in N$, respecting $v_{i}(0)=0$ and the asymptotic conditions respected by $v$. We say that there is a suitable host if there is a $i_{s} \in N$ such that $v_{i_{s}}^{\prime \prime \prime}>0, v_{i_{s}}^{\prime}=\min _{i}\left\{v_{i}^{\prime}\right\}$ and $v_{i_{s}}^{\prime \prime}=\min _{i}\left\{v_{i}^{\prime \prime}\right\}$.

Proposition 6. All the previous results hold under a non-parametrized set of indirect utilities as long as there is a suitable host.

In the parametrized case, it is evident that the community with the lowest $d$ meets the condition for being the suitable host. Proposition 6 reveals that the existence of the suitable host, rather than the parametrization, is a sufficient condition to make the mechanism work.

Proposition 6 leaves open the question on how the mechanism works when the suitable host assumption is not met. We provide a hint to the answer to this question. To do that, we consider the simplest parametrization that allows for the inexistence of a suitable host. We let $v_{i}(q):=d_{i}^{h} v(q) \mathbb{1}_{\{q<0\}}+d_{i}^{b} v(q) \mathbb{1}_{\{q \geq 0\}}$ for each $q$. Here, the ordering of $d_{i}^{h}$ ranks the communities' disutility for being the host, while the ordering of $d_{i}^{b}$ ranks the communities' benefits for being a buyer. Without loss of generality, we impose that $d_{1}^{b}<d_{2}^{b}<\cdots<d_{n}^{b}$.

We explore this scenario using a simulation with three communities. We set $d_{1}^{h}<d_{3}^{h}$ and $d_{1}^{b}<d_{3}^{b}$, with these parameters being fixed; while allowing $d_{2}^{h}$ and $d_{2}^{b}$ to vary. Therefore, the pair $\left(d_{2}^{h}, d_{2}^{b}\right)$ is defined in $\mathbb{R}_{++} \times\left(d_{1}^{b}, \infty\right)$. We are interested in the case where $d_{2}^{h}<d_{1}^{h}$ (otherwise, community 1 would be the suitable host). 


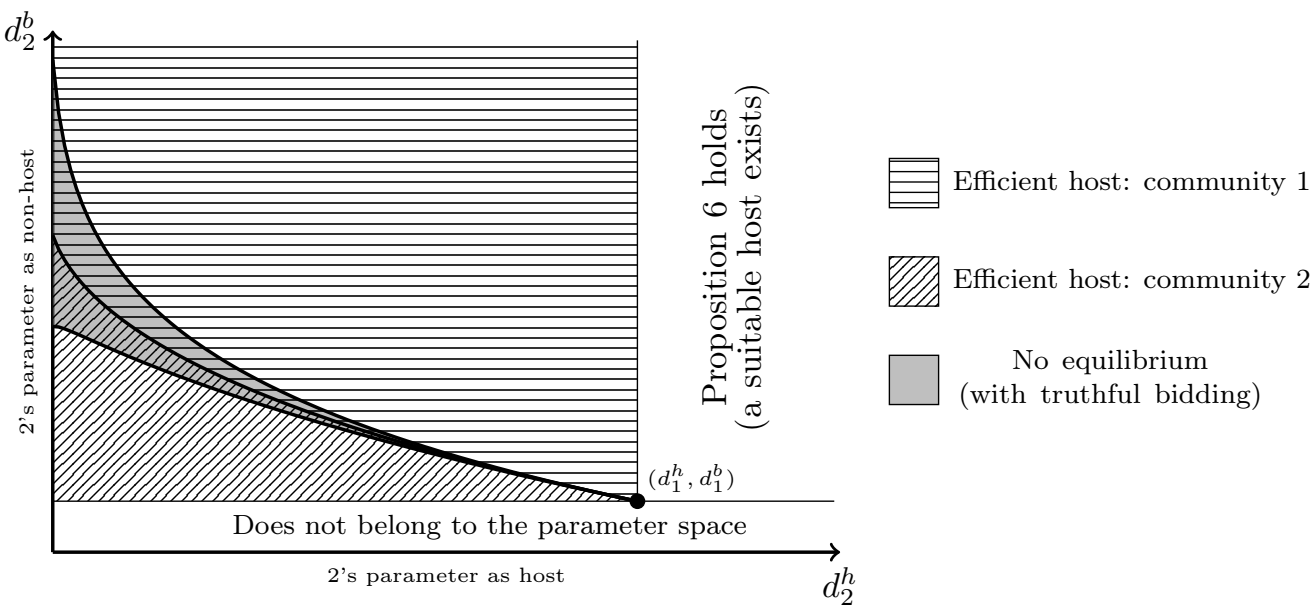

Figure 6: Mechanism outcomes (with truthful bidding) when a suitable host does not exist. We set $d_{1}^{h}=d_{1}^{b}=1, d_{3}^{h}=d_{3}^{b}=5, v=1-e^{-(\cdot)}$, and we assume there is no building cost for the facility. Each point of this plane represents a pair of parameters of community 2 .

Figure 6 depicts the mechanism performance for a particular set of parameters. ${ }^{19}$ Each point represents a pair of community 2's parameters $\left(d_{2}^{h}, d_{2}^{b}\right)$. The hatched region displays the set where no suitable host exists. In particular, the horizontally-hatched region corresponds to the set of parameters making community 1 being the efficient host. The diagonallyhatched region defines an analogous set for community 2. The gray-shaded region, which we call the fail-region, maps the parameter configurations for which the mechanism fails to assign the most efficient allocation when communities bid truthfully. More specifically, the mechanism does not provide an equilibrium with truthful bidding when $\left(d_{2}^{h}, d_{2}^{b}\right)$ is in the fail-region.

This figure's most relevant characteristic is the small size of the fail-region relative to the hatched area. Hence, our simulations suggest that the mechanism is likely to provide efficient allocations in the region where the suitable host does not exist. More importantly, following our arguments from Section 3.1, we believe that the negative correlations between $d_{2}^{h}$ and $d_{2}^{b}$, representing the hatched area, are unlikely to occur. By contrast, $d_{i}^{h}$ and $d_{i}^{b}$ are typically falling into the area where Proposition 6 holds. Hence, the likelihood of falling into the fail-region area is small compared to the rest of the parameter space. We thus conjecture that our mechanism works in most cases.

\footnotetext{
${ }^{19}$ We have tested a plethora of sets of parameters, and they all yield similar results.
} 


\section{Conclusion}

Not every Lindahl outcome is equal when the location of a public project is ex-ante undefined. Whereas all of them are locally efficient, we devised a mechanism that selects the only globally efficient Lindahl allocation in providing a noxious facility.

The functioning of our mechanism comprises two stages: a market-like and an auctionlike step. The market embedded in the mechanism ensures, in equilibrium, that the total price offered by the buyers matches the price demanded by the host. In this step, using a geometric mean for the aggregation of the desired facility size grants an optimal quantity. This combination of prices and quantities ensures a Lindahl allocation. On the other hand, the embedded second-price auction aims at selecting as a host the community that benefits the most from hosting the facility (via the requested compensation), which also grants the globally efficient facility size. This outcome is guaranteed if all the communities truthfully reveal their additional earnings from role switching (i.e., becoming the host instead of being a buyer), a non-dominated strategy.

Although the selection and siting capabilities of the proposed mechanism constitute our main contribution, it does not always deliver the first best outcome in equilibrium for two reasons. First, from the standpoint of the communities, some equilibria are not budget balanced. Second, even if all equilibria dominate the status quo, the mechanism cannot ensure the optimal Lindahl allocation when communities do not bid truthfully.

Besides achieving efficiency, the mechanism displays two essential features for practical applications. First, the informational structure is realistic. Each community is required to know its own preferences, but not other communities' preferences. Second, the message submitted by each community does not become more complex as the number of participants in the mechanism or the range size of the facility increase.

Markets and auctions are traditionally seen as substitute mechanisms for solving allocation problems. Here, we rather combine them within our mechanism in a complementary manner. This approach may shed light on how to tackle other economic problems in which local optima coexist, and selection is crucial in understanding welfare implications.

\section{References}

Ambec, S. and Kervinio, Y. (2016). Cooperative decision-making for the provision of a locally undesirable facility. Social Choice and Welfare, 46(1):119-155. 
Chen, Y. (2002). A family of supermodular Nash mechanisms implementing Lindahl allocations. Economic Theory, 19(4):773-790.

Jackson, M. O. and Swinkels, J. M. (2005). Existence of equilibrium in single and double private value auctions. Econometrica, 73(1):93-139.

Kleindorfer, P. R. and Sertel, M. R. (1994). Auctioning the provision of an indivisible public good. Journal of Economic Theory, 64(1):20-34.

Kunreuther, H., Kleindorfer, P., Knez, P. J., and Yaksick, R. (1987). A compensation mechanism for siting noxious facilities: theory and experimental design. Journal of Environmental Economics and Management, 14(4):371-383.

Kunreuther, H. and Kleindorfer, P. R. (1986). A sealed-bid auction mechanism for siting noxious facilities. American Economic Review, 76(2):295-299.

Laurent-Lucchetti, J. and Leroux, J. (2010). Lindahl prices solve the nimby problem. Economics Bulletin, 30(3):2457-2463.

Laurent-Lucchetti, J. and Leroux, J. (2011). Choosing and sharing. Games and Economic Behavior, 73(1):296-300.

Meyerhoff, J., Ohl, C., and Hartje, V. (2010). Landscape externalities from onshore wind power. Energy Policy, 38(1):82-92.

Minehart, D. and Neeman, Z. (2002). Effective siting of waste treatment facilities. Journal of Environmental Economics and Management, 43(2):303-324.

Olson, M. (1965). Logic of collective action: Public goods and the theory of groups. Cambridge, MA: Harvard University Press.

O'Sullivan, A. (1993). Voluntary auctions for noxious facilities: incentives to participate and the efficiency of siting decisions. Journal of Environmental Economics and Management, 25(1):S12-S26.

Perez-Castrillo, D. and Wettstein, D. (2002). Choosing wisely: A multibidding approach. American Economic Review, 92(5):1577-1587.

Popper, F. J. (1983). LULUs: Locally unwanted land uses. Resources, 73. 
Sakai, T. (2012). Fair waste pricing: an axiomatic analysis to the NIMBY problem. Economic Theory, 50(2):499-521.

Schively, C. (2007). Understanding the NIMBY and LULU phenomena: Reassessing our knowledge base and informing future research. Journal of Planning Literature, 21(3):255266.

Van Essen, M. and Walker, M. (2017). A simple market-like allocation mechanism for public goods. Games and Economic Behavior, 101:6-19.

Waehrer, K. (2003). Hazardous facility siting when cost information is private: an application of multidimensional mechanism design. Journal of Public Economic Theory, 5(4):605-622.

Zerrahn, A. (2017). Wind power and externalities. Ecological Economics, 141:245-260. 


\section{Appendix: Lemmata and proofs of propositions}

\section{Proof of Proposition 1}

Proof. For a fixed facility location $j \in L_{I}$, the social planner's solution satisfies Equation 1. Let $\phi(d)$ be the optimal provided level of $Z$ when it is hosted by a community with parameter $d$ while keeping $\sum_{i} d_{i}$ constant. Define the map

$$
S(d):=v(\phi(d)) \sum_{i} d_{i}+d(v(-\phi(d))-v(\phi(d)))-c \phi(d)
$$

for each $d$ whenever it is well-defined. Notice that $S\left(d_{j}\right)$ is the maximum surplus, constrained to install $Z$ in $j$. By the envelope theorem,

$$
\frac{\mathrm{d} S\left(d_{j}\right)}{\mathrm{d} d}=v\left(-Q^{L_{i}}\right)-v\left(Q^{L_{i}}\right)<0 .
$$

Therefore, as we decrease $j$, we increase the surplus. As a result, the maximum surplus occurs when community 1 hosts $Z$ and provides a quantity $Q^{L_{1}}$.

Lemma 1. If $\boldsymbol{s}$ is an equilibrium of $\mathcal{M}$, then the provision level of $Z, \hat{Q}(s)$, is positive.

Proof. Suppose, by contradiction, that $\hat{Q}(\boldsymbol{s})=0$. Then the payoff of $\hat{i}(\boldsymbol{s})$ is $-r$. If $\hat{i}(\boldsymbol{s})$ deviates its bid to 0 and its price as host to $p_{i}^{h}+\epsilon$ for any $i \neq \hat{i}$ and $\epsilon>0, \hat{i}(s)$ would not be the host anymore. As a buyer, $\hat{i}(\boldsymbol{s})$ could deviate its submitted quantity to a small enough value, ensuring a utility greater than $-r$. Therefore, $s$ cannot be an equilibrium.

Lemma 2. If $\boldsymbol{s}$ is an equilibrium of $\mathcal{M}$, then $p_{\hat{i}(\boldsymbol{s})}^{h}>0$ and $p_{i}^{b}>0$ for all $i \neq \hat{i}(\boldsymbol{s})$.

Proof. By Lemma 1, the implementation rule is respected under strategy $s$. Suppose, by contradiction, that $p_{i}^{b}=0$ for some $i \neq \hat{i}(\boldsymbol{s})$, so $i$ 's payoff as a function of the provision level of $Z$ is $u_{i}(\cdot, 0)$. Since the function $u_{i}(\cdot, 0)$ is strictly increasing, $u_{i}(2 \hat{Q}(\boldsymbol{s}), 0)>u_{i}(\hat{Q}(\boldsymbol{s}), 0)$. But $i$ can deviate its submitted quantity to generate a provision level of $Z$ equal to $2 \hat{Q}(s)$. This contradicts the fact that $s$ is an equilibrium.

Suppose, also by contradiction, that $p_{\hat{i}(s)}^{h}=0$. Notice that $u_{i}(-\hat{Q}(s), 0)<0$ and that $u_{i}(-\hat{Q}(\boldsymbol{s}), 0)<u_{i}(-\hat{Q}(\boldsymbol{s}) / 2,0)$. But $\hat{i}(\boldsymbol{s})$ could deviate its submitted quantity to generate a provision level of $Z$ equal to $\hat{Q}(s) / 2$, contradicting that $s$ is an equilibrium.

Lemma 3. If $\boldsymbol{s}$ is an equilibrium with project implementation, then $p_{\hat{i}(\boldsymbol{s})}^{h}+c=\sum_{i \neq \hat{i}(\boldsymbol{s})} p_{j}^{b}$. 
Proof. Since, by Lemma 1, the project is implemented, we have $p_{\hat{i}(\boldsymbol{s})}^{h}+c \leq \sum_{j \neq \hat{i}(\boldsymbol{s})} p_{j}^{b}$. Suppose, by contradiction, that $\epsilon>0$ is a slacking variable, so $p_{\hat{i}(\boldsymbol{s})}^{h}+c=\sum_{j \neq \hat{i}(\boldsymbol{s})} p_{j}^{b}+\epsilon$. In this case, community $i \neq \hat{i}(\boldsymbol{s})$ could deviate its price as buyer to $p_{i}^{b}-\epsilon / 2$, keeping the implementation of the project at the same provision level, while paying $\hat{T}_{i}(s)-\frac{\epsilon}{2}(1-$ $\left.\frac{1}{2(n-1)}\right) \hat{Q}(s)<\hat{T}_{i}(\boldsymbol{s})$. Therefore, $s_{i}$ cannot be a maximizing strategy for $i$ given $\boldsymbol{s}_{-i}$.

\section{Proof of Proposition 2}

Proof. Recall that $v^{\prime}$ is continuous, strictly decreasing, and (due to the asymptotic conditions) its image is $\mathbb{R}$. Thus, we can apply the Intermediate Value Theorem to deduce that, for any $p>0$ and $d>0$, there is a unique real $q$ satisfying $p=d v^{\prime}(q)$. Since, by Lemma 2 , the operating prices of $s$ are positive, individual solutions must respect the first-order conditions $p_{i}^{b}=d_{i} v^{\prime}(\hat{Q}(\boldsymbol{s}))$ for $i \neq \hat{i}(\boldsymbol{s})$, and $p_{\hat{i}(\boldsymbol{s})}^{h}=d_{\hat{i}(\boldsymbol{s})} v^{\prime}(-\hat{Q}(\boldsymbol{s}))$. In addition, by Lemma 3, we have $p_{\hat{i}(\boldsymbol{s})}^{h}+c=\sum_{j \neq \hat{i}(\boldsymbol{s})} p_{j}^{b}$. This means that $\tilde{\mathcal{M}}(\boldsymbol{s})$ being a Lindahl- $\hat{i}(\boldsymbol{s})$ is a necessary condition for $s$ to be an equilibrium.

Lemma 4. For each $x \geq 0$, the inequality $d_{i} v(-x)-d_{i} v^{\prime}(-x) x>d_{i} v(x)-d_{i} v^{\prime}(x) x$ holds.

Proof. Define $\phi$ as a function mapping each $x>0$ to $\phi(x)=d_{i} v(-x)-d_{i} v(x)$. Since, by assumption, $v^{\prime \prime \prime}>0$, then $\phi^{\prime \prime}(x)=d_{i} v^{\prime \prime}(-x)-d_{i} v^{\prime \prime}(x)<0$ and $\phi$ is concave. But if $\phi$ is concave, we must have $\phi(y)-\phi(x)-\phi^{\prime}(x)(x-y)<0$ for each $x, y \in \mathbb{R}_{+}$. In particular, if $y=0$ we have $-\phi(x)-\phi^{\prime}(x) x<0$. Rearranging this inequality we have the desired result.

Lemma 5. Let $p_{1}^{b}=d_{1} v^{\prime}\left(Q^{L_{1}}\right)$ and suppose that, for some $i \geq 2, p_{1}^{L_{1}}-p_{i}^{L_{1}}+p_{1}^{b}>d_{i} v^{\prime}(0)$ holds. Define $p_{i}^{h}:=p_{1}^{L_{1}}-p_{i}^{L_{1}}+p_{1}^{b}$ and $\tilde{Q}_{i}$ such that $d_{i} v^{\prime}\left(-\tilde{Q}_{i}\right)=p_{i}^{h}$. Then $0<\tilde{Q}_{i}<Q^{L_{1}}$ and we have

$$
d_{1} v\left(-Q^{L_{1}}\right)-d_{1} v\left(Q^{L_{1}}\right)-d_{i} v\left(-\tilde{Q}_{i}\right)+d_{i} v\left(Q^{L_{1}}\right)>\left(p_{1}^{L_{1}}-p_{i}^{L_{1}}+p_{1}^{b}\right)\left(\tilde{Q}_{i}-Q^{L_{1}}\right)
$$

Proof. The positiveness of $\tilde{Q}_{i}$ holds trivially from $p_{i}^{h}>d_{i} v^{\prime}(0)=\bar{p}_{i}$. To show that $\tilde{Q}_{i}<Q^{L_{1}}$, notice first that $d_{1}<d_{i}$ implies $p_{1}^{b}<p_{i}^{L_{1}}$. In turn, this implies that $p_{i}^{h}<p_{1}^{L_{1}}$ and $p_{i}^{h} / d_{i}<p_{1}^{L_{1}} / d_{1}$. Because $v^{\prime}$ is strictly decreasing we have

$$
-\tilde{Q}_{i}=v^{\prime-1}\left(p_{i}^{h} / d_{i}\right)>v^{\prime-1}\left(p_{1}^{L_{1}} / d_{1}\right)=-Q^{L_{1}}
$$

or $\tilde{Q}_{i}<Q^{L_{1}}$. 
Define, for each $x \in\left[0, Q^{L_{1}}-\tilde{Q}_{i}\right]$, the following two maps

$$
F(x)=d_{1} v\left(-\left(\tilde{Q}_{i}+x\right)\right)-d_{1} v\left(\tilde{Q}_{i}+x\right)-d_{i} v\left(-\tilde{Q}_{i}\right)+d_{i} v\left(\tilde{Q}_{i}+x\right)
$$

and

$$
G(x)=\left(p_{1}^{L_{1}}-p_{i}^{L_{1}}+p_{1}^{b}\right)\left(\tilde{Q}_{i}-\left(\tilde{Q}_{i}+x\right)\right) .
$$

We want to prove that $F\left(Q^{L_{1}}-\tilde{Q}_{i}\right)>G\left(Q^{L_{1}}-\tilde{Q}_{i}\right)$. Notice that $G(0)=0$ and that, after rearranging $F(0)$,

$$
F(0)=\left(d_{i}-d_{1}\right)\left(v\left(\tilde{Q}_{i}\right)-v\left(-\tilde{Q}_{i}\right)\right)>0
$$

so $F(0)>G(0)$.

Also, if $x \in\left[0, Q^{L_{1}}-\tilde{Q}_{i}\right]$, since $v$ is concave we have

$$
d_{1} v^{\prime}\left(-Q^{L_{1}}\right)=p_{1}^{L_{1}}>d_{1} v^{\prime}\left(-\left(\tilde{Q}_{i}+x\right)\right)
$$

and

$$
0<\left(d_{i}-d_{1}\right)\left(v^{\prime}\left(\tilde{Q}_{i}+x\right)-v^{\prime}\left(Q^{L_{1}}\right)\right)=d_{i} v^{\prime}\left(\tilde{Q}_{i}+x\right)-p_{i}^{L_{1}}-d_{1} v^{\prime}\left(\tilde{Q}_{i}+x\right)+p_{1}^{b}
$$

Adding up the two previous inequalities and rearranging them yields

$$
-d_{1} v^{\prime}\left(-\left(\tilde{Q}_{i}+x\right)\right)-d_{1} v^{\prime}\left(\tilde{Q}_{i}+x\right)+d_{i} v^{\prime}\left(\tilde{Q}_{i}+x\right)>p_{i}^{L_{1}}-p_{1}^{L_{1}}-p_{1}^{b} .
$$

This inequality can be written as $F^{\prime}(x)>G^{\prime}(x)$, so

$$
\int_{0}^{Q^{L_{1}-\tilde{Q}_{i}}} F^{\prime}(x) \mathrm{d} x>\int_{0}^{Q^{L_{1}-\tilde{Q}_{i}}} G^{\prime}(x) \mathrm{d} x
$$

and applying the fundamental theorem of calculus gives us

$$
F\left(Q^{L_{1}}-\tilde{Q}_{i}\right)-F(0)>G\left(Q^{L_{1}}-\tilde{Q}_{i}\right)-G(0) .
$$

Since $F(0)>G(0)$ we have $F\left(Q^{L_{1}}-\tilde{Q}_{i}\right)>G\left(Q^{L_{1}}-\tilde{q}_{i}\right)$, concluding the proof.

\section{Proof of Proposition 3}

Proof. First, we prove that $s:=s^{\star}$ is an equilibrium, and then we prove the robustness on coalitions excluding community 1 . To prove that $s$ is an equilibrium, it suffices to show that 
1. $b_{1}>b_{i}$ for $i \geq 2$ 2. community 1 has no incentives to deviate from the role of host, and 3. community $i$ for $i \geq 2$ has no incentives to deviate from the role of buyer.

The first and third steps are easier to show for $i \geq 2$ when $p_{i}^{h}=d_{i} v^{\prime}(0)$ and, thus, $b_{i}=0$. By Lemma $4, b_{1}$ is strictly positive, so $b_{1}>b_{i}=0$. Besides, if $i$ deviates its bid do $\tilde{b}_{i}$ such that $\tilde{b}_{i}>b_{1}$, then $i$ would become the host. A price as host submitted by $i$ as a deviating strategy can lead to two possible outcomes. If this price respects the implementation rule, then it is lower than the no-trade price of $i$, conferring this community a negative payoff. Otherwise, $i$ 's payoff would be $-r-b_{1}$. Any of these outcomes would be worse for $i$ than the positive payoff obtained from being a buyer under Lindahl-1. We conclude that, when $i$ is such that $p_{i}^{h}=d_{i} v^{\prime}(0)$, conditions $\mathbf{1}$. and $\mathbf{3}$. are met.

We now consider the case when $p_{i}^{h}>d_{i} v^{\prime}(0)$. Lemma 5 shows $\mathbf{1}$.. show 2 ., recall that the payoff of community 1 as the host is $d_{1} v\left(-Q^{L_{1}}\right)+p_{1}^{L_{1}} Q^{L_{1}}-b_{\hat{j}(s)}$, while as a buyer it would be at most $d_{1} v\left(Q^{L_{1}}\right)-p_{1}^{b} q^{L_{1}}$. However, note that

$$
\begin{aligned}
d_{1} v\left(-Q^{L_{1}}\right)+p_{1}^{L_{1}} Q^{L_{1}}-b_{\hat{j}(\boldsymbol{s})} & >d_{1} v\left(-Q^{L_{1}}\right)+p_{1}^{L_{1}} Q^{L_{1}}-b_{1} \\
& =d_{1} v\left(Q^{L_{1}}\right)-p_{1}^{b} L^{L_{1}},
\end{aligned}
$$

so community 1 does not have incentives to deviate from its host position.

We prove 3. To be a host, community $i$ has to deviate its bid to some $\tilde{b}_{i}$ such that $\tilde{b}_{i}>b_{1}$. In this case, the second-highest bid is $b_{1}$ and community $i$ would get at most $d_{i} v\left(-\tilde{Q}_{i}\right)+p_{i}^{h} \tilde{Q}_{i}-b_{1}$. But Lemma 5 (after rearranging terms) yields

$$
d_{i} v\left(-\tilde{Q}_{i}\right)+p_{i}^{h} \tilde{Q}_{i}-b_{1}<d_{i} v\left(Q^{L_{1}}\right)-p_{i}^{L_{1}} Q^{L_{1}} .
$$

Since the left-hand side is $i$ 's payoff as a buyer, community $i$ has no incentives to deviate from $s_{i}$, proving that $s$ is an equilibrium.

Now we show the robustness of $s$ to coalitions. Let $I \subseteq N \backslash\{1\}$ be a set (i.e., a coalition) with at least two elements and let $s_{-I}$ be $s$ without the components in $I$. The equilibrium $s$ is robust to the coalition $I$ if, and only if, the coalition under $s_{-I}$ cannot generate a surplus larger than the one obtained in $s$. We have two possible coalitional deviations: (i) each community in $I$ remains a buyer; and (ii) a community in $I$ becomes the host. We let $\tilde{p}_{i}^{b}$ be the new price as buyer of community $i \in I$ after deviating from $s$ and $\tilde{p}_{i}^{h}$ the analogous for the price as host. Recall that, for both cases, if $Z$ is not implemented, this would give a null surplus for the coalition, which would make the coalition worse off. So, if there is a strategy improving the coalition's surplus, it has to imply a positive provision of $Z$. 
In case (i), the problem $I$ solves is to maximize

$$
\sum_{i \in I}\left(d_{i} v(\tilde{Q})-\tilde{p}_{i}^{b} \tilde{Q}\right)=v(\tilde{Q}) \sum_{i \in I} d_{i}+\tilde{Q} \sum_{i \in I} \tilde{p}_{i}^{b}
$$

on variables with a tilde, subject to $\sum_{i \in I} \tilde{p}_{i}^{b} \geq \sum_{i \in I} p_{i}^{L_{1}}$. This constraint ensures the implementation of $Z$. At the maximum, the restriction is binding, so we can rewrite the coalition problem as to maximize $v(\tilde{Q}) \sum_{i \in I} d_{i}+\tilde{Q} \sum_{i \in I} p_{i}^{L_{1}}$ on $\tilde{Q}$. But this is a strictly concave function on $\tilde{Q}$, so there is a unique maximum respecting the first-order condition. We notice that $Q^{L_{1}}$ solves the FOC. Therefore, strategy $s$ provides already the maximum surplus for $I$.

In case (ii), let $j \in I$ be the host. The problem coalition $I$ solves is to maximize

$$
d_{j} v(-\tilde{Q})+v(\tilde{Q}) \sum_{i \in I \backslash\{j\}} d_{i}+\tilde{Q}\left(-\tilde{p}_{j}^{h}+\sum_{i \in I \backslash\{j\}} \tilde{p}_{i}^{b}\right)-b_{1}
$$

on variables with a tilde, subject to $\sum_{i \in I \backslash\{j\}} \tilde{p}_{i}^{b}-\tilde{p}_{j}^{h} \geq \sum_{i \in I \backslash\{j\}} p_{i}^{L_{1}}+p_{j}^{L_{1}}-p_{1}^{L_{1}}-p_{1}^{b}$. As in case (i), this constraint implies the implementation of $Z$ and any solution has to respect it with equality. Therefore, we can rewrite the coalition's problem and apply maxima properties to obtain

$$
\begin{aligned}
& \max _{\tilde{Q}>0}\left\{\sum_{i \in I \backslash\{j\}}\left(d_{i} v(\tilde{Q})-p_{j}^{L_{1}} \tilde{Q}\right)+d_{j} v(-\tilde{Q})+\left(p_{1}^{L_{1}}-p_{j}^{L_{1}}+p_{1}^{b}\right) \tilde{Q}-b_{1}\right\} \\
\leq & \sum_{i \in I \backslash\{j\}} \max _{\tilde{Q}>0}\left\{d_{i} v(\tilde{Q})-p_{j}^{L_{1}} \tilde{Q}\right\}+\max _{\tilde{Q}>0}\left\{d_{j} v(-\tilde{Q})+\left(p_{1}^{L_{1}}-p_{j}^{L_{1}}+p_{1}^{b}\right) \tilde{Q}-b_{1}\right\} \\
\leq & \sum_{i \in I \backslash\{j\}}\left(d_{i} v\left(Q^{L_{1}}\right)-p_{j}^{L_{1}} Q^{L_{1}}\right)+g_{j}^{h}\left(\boldsymbol{s}_{-j}\right)-b_{1} \\
< & \sum_{i \in I \backslash\{j\}} g_{i}^{b}\left(\boldsymbol{s}_{i}\right)+g_{j}^{b}\left(\boldsymbol{s}_{-j}\right)
\end{aligned}
$$

where the last inequality is a direct application of Lemma 5. But $\sum_{i \in I \backslash\{j\}} g_{i}^{b}\left(\boldsymbol{s}_{i}\right)+g_{j}^{b}\left(\boldsymbol{s}_{-j}\right)$ is the surplus $I$ gets under strategy $s$. Thus, $I$ is strictly better off under $s$ than under any other strategy to which coalition $I$ can deviate. This completes the proof.

Lemma 6. For $i \geq 2$, let $Q>0$ and $\tilde{q}$ be such that

$$
d_{i} v^{\prime}(\tilde{q})-d_{1} v^{\prime}(Q)=d_{1} v^{\prime}(-\tilde{q})-d_{i} v^{\prime}(-Q)
$$


Then $\tilde{q} \geq Q$ and

$$
d_{1} v(-\tilde{q})+\left(d_{i} v^{\prime}(-Q)+d_{i} v^{\prime}(\tilde{q})-d_{1} v^{\prime}(Q)\right) \tilde{q}>d_{1} v(Q)-d_{1} v^{\prime}(Q) Q>0
$$

Proof. Suppose, by contradiction, that $\tilde{q}<Q$. Since $v^{\prime}$ is decreasing and $d_{i}>d_{1}$, we have $d_{i} v^{\prime}(\tilde{q})-d_{1} v^{\prime}(Q)>0$ and $d_{1} v^{\prime}(-\tilde{q})-d_{i} v^{\prime}(-Q)<0$. Hence, the first equality would not hold, contradicting $\tilde{q}<Q$.

We can rewrite the left-hand side of the inequality as $d_{1} v(-\tilde{q})+d_{1} v^{\prime}(-\tilde{q}) \tilde{q}$. Defining, for each $x>0$, the function $\phi(x)=d_{1} v(-x)+d_{1} v^{\prime}(-x) x$, we observe that $\phi^{\prime}(x)=-d_{1} v^{\prime \prime}(-x) x>0$, so $\phi$ is strictly increasing. Thus we have

$$
\begin{aligned}
d_{1} v(-\tilde{q})+d_{1} v^{\prime}(-\tilde{q}) \tilde{q} & >d_{1} v(-Q)+d_{1} v^{\prime}(-Q) Q \\
& >d_{1} v(Q)-d_{1} v^{\prime}(Q) Q>0
\end{aligned}
$$

where we use Lemma 4 to get the second inequality and the fact that $d_{1} v-d_{1} v^{\prime}$ Id is increasing and equal to 0 at 0 to get the third inequality.

Lemma 7. Suppose $i \in L_{I}, i \geq 2$ and, for any $y \geq 0$, let $\tilde{q}_{1}$ and $\tilde{q}_{i}$ be functions respecting $d_{i} v^{\prime}\left(\tilde{q}_{i}(y)\right)=y$ and $d_{1} v^{\prime}\left(-\tilde{q}_{1}(y)\right)=p_{i}^{L_{i}}+y-p_{1}^{L_{i}}$. Then

$d_{i} v\left(-Q^{L_{i}}\right)+p_{i}^{L_{i}} Q^{L_{i}}-d_{i} v\left(\tilde{q}_{i}(y)\right)+y \tilde{q}_{i}(y)<d_{1} v\left(-\tilde{q}_{1}(y)\right)+\left(p_{i}^{L_{i}}+y-p_{1}^{L_{i}}\right) \tilde{q}_{1}(y)-d_{1} v\left(Q^{L_{i}}\right)+p_{1}^{L_{i}} Q^{L_{i}}$.

Proof. We prove this Lemma in two steps. First, we show that the inequality is the tightest when $y=p$ such that $\tilde{q}_{i}(p)=\tilde{q}_{1}(p)$. Then, we show the main inequality.

First, notice that functions $\tilde{q}_{i}$ and $\tilde{q}_{1}$ are well-defined, continuous and monotonic functions. Thus, we can apply the Implicit Function Theorem to find that $\tilde{q}_{1}^{\prime}=-1 / d_{1} v^{\prime \prime}\left(-\tilde{q}_{1}\right)>0$ and $\tilde{q}_{i}^{\prime}=1 / d_{i} v^{\prime \prime}\left(\tilde{q}_{i}\right)<0$. Observe that the inequality is the tightest when the function $\phi$, defined for each $y>0$ by

$$
\phi(y)=-d_{i} v\left(\tilde{q}_{i}(y)\right)+\tilde{q}_{i}(y) y-d_{1} v\left(-\tilde{q}_{1}(y)\right)-\left(p_{i}^{L_{i}}+y-p_{1}^{L_{i}}\right) \tilde{q}_{1}(y)
$$

attains its maximum. Differentiating $\phi$, and using the fact that $d_{1} v^{\prime}\left(-\tilde{q}_{1}(y)\right)=p_{i}^{L_{i}}+y-p_{1}^{L_{i}}$ and $d_{i} v^{\prime}\left(\tilde{q}_{i}(y)\right)=y$, we obtain $\phi^{\prime}=\tilde{q}_{i}-\tilde{q}_{1}$. Differentiating once more we get $\phi^{\prime \prime}=\tilde{q}_{i}^{\prime}-\tilde{q}_{1}^{\prime}$. Since $\tilde{q}_{i}^{\prime}<0$ and $\tilde{q}_{1}^{\prime}>0$, we have that $\phi^{\prime \prime}<0$. This means that $\phi$ attains its maximum at $p$ such that $\phi^{\prime}(p)=0$, which is the same as $\tilde{q}_{i}(p)=\tilde{q}_{1}(p)$. It follows that we only need to prove the inequality for the case when $y=p$. We call $\tilde{q}_{1}(p)=: \tilde{q}$. 
To prove the second part, we observe first that $d_{i} v^{\prime}(\tilde{q})-d_{1} v^{\prime}\left(Q^{L_{i}}\right)=d_{1} v^{\prime}(-\tilde{q})-d_{i} v^{\prime}\left(-Q^{L_{i}}\right)$, so we can apply Lemma 6 to conclude that $\tilde{q} \geq Q^{L_{i}}$. Define for all $x \in\left[0, \tilde{q}-Q^{L_{i}}\right]$ the following two maps

$$
F(x)=d_{i} v\left(-Q^{L_{i}}\right)-d_{i} v\left(Q^{L_{i}}+x\right)-d_{1} v\left(-Q^{L_{i}}-x\right)+d_{1} v\left(Q^{L_{i}}\right)
$$

and

$$
G(x)=\left(p_{i}^{L_{i}}-p_{1}^{L_{i}}\right) x .
$$

We want to prove that $F\left(\tilde{q}-Q^{L_{i}}\right)<G\left(\tilde{q}-Q^{L_{i}}\right)$. Notice that $G(0)=0$ and that, after rearranging $F(0)$,

$$
F(0)=\left(d_{i}-d_{1}\right)\left(v\left(-Q^{L_{i}}\right)-v\left(Q^{L_{i}}\right)\right)<0,
$$

so $F(0)<G(0)$.

In addition, if $x \in\left(0, \tilde{q}-Q^{L_{i}}\right]$ and since $v^{\prime \prime}<0$, we have $-d_{i} v^{\prime}\left(Q^{L_{i}}+x\right)<-d_{i} v^{\prime}(\tilde{q})=-p$ and $d_{1} v^{\prime}\left(-Q^{L_{i}}-x\right)<d_{1} v^{\prime}(-\tilde{q})=p_{i}^{L_{i}}+p-p_{1}^{L_{i}}$. Adding these two inequalities gives

$$
-d_{i} v^{\prime}\left(Q^{L_{i}}+x\right)+d_{1} v^{\prime}\left(-Q^{L_{i}}-x\right)<p_{i}^{L_{i}}-p_{1}^{L_{i}}
$$

This last inequality is the same as $F^{\prime}(x)<G^{\prime}(x)$, hence

$$
\int_{0}^{\tilde{q}-Q^{L_{i}}} F^{\prime}(x) \mathrm{d} x<\int_{0}^{\tilde{q}-Q^{L_{i}}} G^{\prime}(x) \mathrm{d} x
$$

and by the fundamental theorem of calculus

$$
F\left(\tilde{q}-Q^{L_{i}}\right)-F(0)<G\left(\tilde{q}-Q^{L_{i}}\right)-G(0) .
$$

We conclude the proof using the fact that $F(0)<G(0)$.

\section{Proof of Proposition 4}

Proof. Suppose, by contradiction, that $s$ is an equilibrium such that $\tilde{\mathcal{M}}(s)$ is a Lindahl- $j$ outcome. First, consider the case when $\Delta_{1}\left(s_{-1}\right)>0$. Applying Lemma 7 it is straightforward to conclude that $\Delta_{1}\left(s_{-1}\right)>\Delta_{j}\left(s_{-j}\right)$. This contradicts $j$ ending up as the host if communities bid their switching valuations.

What remains left to show is that $s$ cannot be an equilibrium, even when $\Delta_{1}\left(s_{-1}\right) \leq 0$. To do that, we need to show that, in this case, all the other $\Delta$ 's under $s$ are non-positive. For each $i \geq 2$, let $\tilde{q}_{i}$ solve $d_{j} v^{\prime}\left(\tilde{q}_{i}\right)=p_{j}^{L_{j}}-p_{i}^{b}+p_{j}^{b}\left(\tilde{q}_{i}\right.$ can be negative) and notice that 
$\tilde{q}_{j}=Q^{L_{j}}$. We recall that $v-v^{\prime}$ Id is increasing and it is 0 at $0, v\left(q^{L_{j}}\right)-v^{\prime}\left(Q^{L_{j}}\right) Q^{L_{j}}>0$, and $v\left(-\tilde{Q}_{i}\right)-\left(v\left(Q^{L_{j}}\right)-v^{\prime}\left(Q^{L_{j}}\right) Q^{L_{j}}\right)<0$. Thus, for each $i \geq 2$, we have ${ }^{20}$

$$
\begin{aligned}
& \Delta_{i}\left(\boldsymbol{s}_{-i}\right)=\left(d_{i} v\left(-\tilde{q}_{i}\right)+\left(p_{j}^{L_{j}}-p_{i}^{b}+p_{j}^{b}\right) \tilde{q}_{i}\right) \mathbb{1}_{\left\{\tilde{q}_{i}>0\right\}}-d_{i}\left(v\left(Q^{L_{j}}\right)-v^{\prime}\left(Q^{L_{j}}\right) Q^{L_{j}}\right) \\
& {\left[d_{1}<d_{i}\right] }<\left(d_{1} v\left(-\tilde{q}_{i}\right)+\left(p_{j}^{L_{j}}-p_{i}^{b}+p_{j}^{b}\right) \tilde{q}_{i}\right) \mathbb{1}_{\left\{\tilde{q}_{i}>0\right\}}-d_{1}\left(v\left(Q^{L_{j}}\right)-v^{\prime}\left(Q^{L_{j}}\right) Q^{L_{j}}\right) \\
& {\left[p_{1}^{b} \leq p_{i}^{b}\right] \quad \leq\left(d_{1} v\left(-\tilde{q}_{i}\right)+\left(p_{j}^{L_{j}}-p_{1}^{b}+p_{j}^{b}\right) \tilde{q}_{i}\right) \mathbb{1}_{\left\{\tilde{q}_{i}>0\right\}}-d_{1}\left(v\left(Q^{L_{j}}\right)-v^{\prime}\left(Q^{L_{j}}\right) Q^{L_{j}}\right) } \\
& \leq\left(d_{1} v\left(-\tilde{q}_{1}\right)+\left(p_{j}^{L_{j}}-p_{1}^{b}+p_{j}^{b}\right) \tilde{q}_{1}\right) \mathbb{1}_{\left\{\tilde{q}_{1}>0\right\}}-d_{1} v\left(Q^{L_{j}}\right)+p_{1}^{L_{j}} Q^{L_{j}}=\Delta_{i}\left(\boldsymbol{s}_{-1}\right) \leq 0 .
\end{aligned}
$$

We just concluded that having $\Delta_{1}\left(s_{-1}\right) \leq 0$ implies that all the switching valuations are null and that $j$ would be better off as a buyer. But this is a feasible outcome for $j$ under $\boldsymbol{s}_{-j}$. To achieve it, $j$ could deviate by submitting its optimal quantity as a buyer, and increasing its price as host until it is not the smallest submitted one. So $s$ cannot be an equilibrium generating a Lindahl- $j$ outcome.

\section{Proof of Proposition 6}

Proof. It suffices to replace $d_{i} v$ by $v_{i}$ and $d_{1} v$ by $v_{i_{s}}$ and repeat the steps of the proofs of some lemmata. We list the lemmata where the suitable host assumption is directly used: Lemma 4 needs $v_{i_{s}}^{\prime \prime \prime}>0$ to hold, while Lemmata 5, 6, and 7 need $v_{i_{s}}^{\prime}=\min _{i}\left\{v_{i}^{\prime}\right\}$ and $v_{i_{s}}^{\prime \prime}=\min _{i}\left\{v_{i}^{\prime \prime}\right\}$ to hold.

\footnotetext{
${ }^{20}$ If $\tilde{q}_{i} \leq 0$, then $d_{i} v\left(-\tilde{q}_{i}\right)+\left(p_{i}^{L_{j}}-p_{i}^{b}+p_{j}^{b}\right) \tilde{q}_{i} \leq 0$, which is also the same as $p_{j}^{L_{j}}-p_{i}^{b}+p_{j}^{b}<d_{i} v^{\prime}(0)$. Hence, in the hypothetical situation of being a host, $i$ could deviate by submitting an arbitrarily small quantity, implying that $g_{i}^{h}\left(\boldsymbol{s}_{-i}\right)=0$.
} 